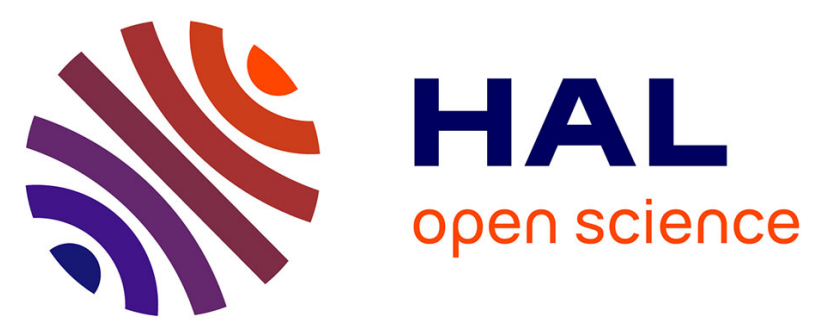

\title{
Effect of puckering motion and hydrogen bond formation on the vibrational circular dichroism spectrum of a flexible molecule: the case of ( $S$ )-1-indanol
}

Katia Le Barbu-Debus, Arne Scherrer, Aude Bouchet, Daniel Sebastiani, Rodolphe Vuilleumier, Anne Zehnacker

\section{To cite this version:}

Katia Le Barbu-Debus, Arne Scherrer, Aude Bouchet, Daniel Sebastiani, Rodolphe Vuilleumier, et al. Effect of puckering motion and hydrogen bond formation on the vibrational circular dichroism spectrum of a flexible molecule: the case of ( S )-1-indanol. Physical Chemistry Chemical Physics, 2018, 20 (21), pp.14635-14646. 10.1039/c8cp01695j . hal-02070261

\section{HAL Id: hal-02070261 \\ https://hal.science/hal-02070261}

Submitted on 22 Jan 2020

HAL is a multi-disciplinary open access archive for the deposit and dissemination of scientific research documents, whether they are published or not. The documents may come from teaching and research institutions in France or abroad, or from public or private research centers.
L'archive ouverte pluridisciplinaire HAL, est destinée au dépôt et à la diffusion de documents scientifiques de niveau recherche, publiés ou non, émanant des établissements d'enseignement et de recherche français ou étrangers, des laboratoires publics ou privés. 


\title{
Effect of Puckering Motion and Hydrogen Bond Formation on the Vibrational Circular Dichroism Spectrum of a Flexible Molecule: the case of (S)-1-Indanol
}

\author{
Katia Le Barbu-Debus, ${ }^{\text {a) }}$ Arne Scherrer, ${ }^{\text {b) c) }}$ Aude Bouchet, ${ }^{\text {a) }}$ \\ Daniel Sebastiani, ${ }^{c}$ Rodolphe Vuilleumier, ${ }^{*}$ b) Anne Zehnacker, * a) \\ a) Institut des Sciences Moléculaires d'Orsay (ISMO), CNRS, Univ. Paris-Sud, \\ Université Paris-Saclay, F-91405 Orsay (France). \\ b) PASTEUR, Département de chimie, École normale supérieure, PSL University, \\ Sorbonne Université, CNRS, 75005 Paris, France \\ c) Martin-Luther-Universität Halle-Wittenberg, Institut für Chemie, von-Danckelmann- \\ Platz 4, 06120 Halle, Germany \\ *e-mail: anne.zehnacker-rentien@u-psud.fr, rodolphe.vuilleumier@ens.fr
}

\begin{abstract}
The influence of flexibility and hydrogen bond formation on the IR absorption and vibrational circular dichroism (VCD) spectrum of a floppy protic molecule, namely, (S)-1-indanol, is studied in both non-polar $\mathrm{CCl}_{4}$ and polar DMSO solvents. The experimental IR absorption and VCD spectra obtained by Fourier transform spectroscopy are interpreted using both static density functional theory (DFT) calculations and first principles molecular dynamics (FPMD) within DFT, using the nuclear velocity perturbation theory (NVPT). Simulation of the spectra based on static optimised geometries is not sufficient in $\mathrm{CCl}_{4}$ and going beyond static calculations is mandatory for satisfactorily reproducing the VCD spectra. The FPMD results obtained in DMSO indicate that (S)-1-indanol is hydrogen-bonded to one DMSO molecule. As a result, static "cluster-in-the-bulk" DFT calculations in which the solutesolvent interaction is modeled as the most stable (S)-1-indanol:DMSO-d6 complexes in a DMSO continuum yield satisfactory agreement with the experiment. Correspondence between experimental and simulated spectra is slightly improved when the VCD spectrum is calculated as the summed contributions of snapshots extracted from FPMD trajectories, due to better sampling of the potentialenergy surface. Finally, NVPT calculations further improve the description of experimental spectra by taking into account higher-energy structures, which are not necessary local minima.
\end{abstract}




\section{Keywords}

Vibrational Circular Dichroism (VCD). Hydrogen bond. FTIR Spectroscopy. Nuclear Velocity Perturbation Theory. Solvation. Molecular dynamics. Cluster formation. Chirality 


\section{Introduction}

Chiroptical spectroscopy has become increasingly important for the measurement of enantiomeric purity, in particular for chiral drugs. ${ }^{1-34}$ In particular vibrational circular dichroism (VCD) combined with quantum chemical calculations has proved to be a unique means of determining the absolute configuration of chiral molecules. ${ }^{5}$ Moreover, VCD is exquisitely sensitive to molecular conformation and provides an indirect, although powerful, means of probing the structure of flexible molecules and its sensitivity to the environment $t^{6-10}$ as well as that of large biopolymers like natural peptides or synthetic foldamers. ${ }^{11,12}$ So far, the most commonly-used theoretical description of VCD relies upon the Magnetic-Field Perturbation (MPF) method developed by Stephens within the frame of the DFT. ${ }^{13}$, ${ }^{14}$ The theoretical description of VCD spectra based on harmonic force field and atomic polar tensors gives very satisfactory results for rigid molecules. ${ }^{14,15}$ However, floppy systems are more challenging, precisely because of the sensitivity of VCD to very small changes in geometry. It has been shown for example that a minor change in the inter-ring angle of binaphthol derivatives leads to inversion of some of the bands observed in the VCD spectrum. ${ }^{16}$ Therefore, complex potential-energy surfaces have to be sampled in a reliable way, in order to precisely locate their minima. Moreover, systems at room temperature explore high-energy regions of the potential-energy surface, which are strongly anharmonic, far from the equilibrium geometry. This combined temperature and anharmonicity effects strongly alter the shape of the VCD spectra. ${ }^{16}$ Indeed, like for IR absorption, static harmonic calculations of VCD spectra decrease in reliability when the conformational flexibility increases. ${ }^{17}$ The effects of anharmonicity have been recently described at the second-order perturbation theory (VPT2) within the frame of the Rayleigh-Schrödinger approach. ${ }^{18,}{ }^{19}$ This method has resulted to excellent agreement between experimental and simulated spectra of the small propylene oxide molecule embedded in a cryogenic matrix. ${ }^{20}$ However, for large and very floppy systems, methods beyond static approaches are required. Force-field based molecular dynamics simulations allow efficient sampling of the potential energy surface. They have been used to determine stable structures of peptide strands whose IR and VCD spectra have been subsequently calculated by DFT methods. ${ }^{21}$

The second bottleneck to reliable modelling of the VCD spectra is the description of the environment, which can considerably modify the position and sign of the bands due to modification of the conformational equilibrium or specific interactions. In some cases, specific interactions result in a chirality transfer from the chiral solute to the non-chiral solvent, or to a molecule in close interaction with the chiral solute in the case of complexes isolated in rare gas matrices. ${ }^{22-28}$ A few attempts have been made recently to use explicit solvent models. In the case of very small peptides like dialanine, explicit solvent molecules are necessary to reproduce the spectral position and shape of the amide stretch I. ${ }^{29}$ In larger peptides, for which intramolecular hydrogen bonding competes efficiently with solvation, the use of explicit solvents seems to be superfluous. ${ }^{30}$ Attempts at mimicking solvation as a sum of clusters of different sizes have also been made. ${ }^{25}$ In this approach, which will be referred to as 
the "cluster-in-a-bulk" approach, the first solvent molecules in strong interaction with the solute are treated explicitly while the rest of the solvent is treated as a continuum. ${ }^{31}$ It yields satisfactory agreement between experimental and simulated spectra and it is able to reproduce chirality transfer from the chiral solute to the achiral solvent, at a low computational cost.

In this context, it is highly desirable to develop efficient methodological approaches that take into account both the influence of temperature and that of the surrounding on the VCD spectra. First principle molecular dynamics (FPMD) methods have been recently developed to account for the solute and the solvent at the same level of theory. ${ }^{32-34}$ In this approach, Nuclear Velocity Perturbation Theory (NVPT) is employed for the treatment of the breakdown of the Born-Oppenheimer approximation, needed to describe the VCD signal. The correction to the Born-Oppenheimer form of the wave function is expressed in terms of the nuclear velocity, in contrast with the MPF method mentioned above, and leads to identical results for rigid benchmark molecules. ${ }^{34}$ By fully accounting for the solvent, the method allows reproducing the signature of chirality transfer from small chiral solutes solute like (R)-propylene oxide to the achiral aqueous solvent. ${ }^{33}$

In what follows, this method is applied to a flexible aromatic alcohol, namely, (S)-1-indanol, shown in Scheme 1. This molecule was chosen for the following reasons: first, it possesses a hydroxyl group that is able to form hydrogen bonds with a proton-accepting solvent, which in turn are expected to influence the shape of the VCD spectrum. Second, (S)-1-indanol shows a large amplitude motion, namely, the puckering of the alicyclic ring. Such inversion motions are indeed important in the context of proline-containing peptides and, like all large-amplitude motions, is challenging. ${ }^{35}$ In (S)-1-indanol, inversion results to two conformers separated by a low-energy barrier, which have been characterised in the gas phase under jet-cooled conditions. ${ }^{36}$ In the most stable one, denoted $\mathrm{I}_{\mathrm{eq}}$ in what follows, the $\mathrm{OH}$ substituent is in pseudo equatorial position relative to the alicyclic ring while in the second one, denoted $\mathrm{I}_{\mathrm{ax}}$, it is in pseudo axial position. The potential-energy surface is very anharmonic and highly sensitive to the environment. The relative population of $\mathrm{I}_{\mathrm{eq}}$ and $\mathrm{I}_{\mathrm{ax}}$ varies with the cooling conditions of the supersonic expansion, and is very sensitive to hydrogen bonding or dimer formation. ${ }^{36-39}$ Indeed, $I_{\text {eq }}$ is favoured in the hydrates, while the dimer contains either $I_{e q}$ or $I_{a x}$ sub-units depending, among other, on the chirality of the monomers.

We will study here (S)-1-indanol in different solvents, both inert in terms of hydrogen bonding $\left(\mathrm{CCl}_{4}\right)$, and acceptor of hydrogen bonds (DMSO). $\mathrm{In} \mathrm{CCl}_{4}$, the main reason for non-satisfactory description of the VCD spectra by standard static methods is expected to be related to the anharmonicity of the potential-energy surface regions that are explored at room temperature. Comparing the experimental VCD spectrum to those calculated within the frame of the double harmonic approximation (MPF method) and those deduced from FPMD (NVPT method) will allow assessing the role of this effect. In a second step, the experimental VCD spectra in DMSO will be analysed by the cluster-in-a-bulk approach including up to two DMSO molecules and compared to the FPMD (NVPT method) results. 
This will allow assessing the validity of the cluster-in-a-bulk approach and give a reliable description of the solute-solvent interaction and its consequences on the shape of the VCD spectrum.

\section{Experimental and Theoretical Methods}

\section{II-A. Experimental Methods}

The vibrational IR absorption and VCD spectra were measured using a FTIR spectrometer Vertex 70 equipped with a VCD module PMA 50 (Bruker). The signal was measured by a MCT IR detector with a $\mathrm{BaF}_{2}$ window, cooled with liquid nitrogen. A spectral resolution of $4 \mathrm{~cm}^{-1}$ was used for both absorption and VCD spectra. The IR radiation was polarized with a linear polarizer then modulated by a $50 \mathrm{kHz} \mathrm{ZnSe}$ photo-elastic modulator (Hinds). A low-pass filter cutting at $2000 \mathrm{~cm}^{-1}$ was added before the linear polarizer to increase the dynamical response of the detector. The signal of the MCT detector was demodulated using a lock-in amplifier (Stanford Research Systems SR 830). The alignment of the spectrometer was carefully verified by checking the mirror-image relation between the VCD spectra of the two enantiomers of camphor $\left(0.3 \mathrm{M}^{\text {in }} \mathrm{CCl}_{4}\right)$ in the same cell as used here. The spectra were measured using $\sim 0.5 \mathrm{M}$ solutions in an adjustable cell (Harricks) with a path length of $156 \mu \mathrm{m}$. No strong dependence of the IR absorption upon concentration was observed. The acquisition time was $\sim 12 \mathrm{~h}$. The spectra shown below are the half difference of those of the two enantiomers. The raw data obtained in $\mathrm{CCl}_{4}$ (spectrum of each enantiomer and baseline) are given in Figure $\mathrm{S} 1$ in the electronic supplementary information (ESI $\dagger$ ). The VCD spectra of (S)-1-indanol were then corrected for baseline deviation by subtracting the solvent spectra recorded under identical conditions. $\mathrm{CCl}_{4}$ and DMSO-d6 solvents as well as enantiopure (R)-1-indanol and (S)-1-indanol were purchased by Aldrich and used without further purification.

\section{II-B. Theoretical methods}

\section{Static approaches}

The angles describing the geometry of (S)-1-indanol are shown in Scheme 1. For all the studied systems, exploration of the potential-energy surface was performed combining the OPLS-2005 force fields with the advanced conformational search implemented in the MacroModel program available in the Schrödinger package. ${ }^{40}$ DMSO-d6 was used for calculating the complexes, in order to take into account the effect of deuteration on the vibrational frequencies. For both isolated molecules and clusters, the conformational search included the solvent permittivity. At the end of this step, all the complexes with energy below $20 \mathrm{kJmol}^{-1}$ were optimized using the B3LYP functional combined to the $6-31++G(d, p)$ basis set. The choice of the method was justified by the fact that it reproduces the energy difference between the two most stable conformers of (S)-1-indanol, as deduced from the Raman or IR intensities in jet-cooled conditions. ${ }^{37}$ The same structures were also optimized including 
D3 empirical corrections for the dispersion. ${ }^{41-43}$ The energy ordering is the same whether dispersion is included or not.

The effect of the solvent on the molecule and clusters geometries was taken into account by the polarizable continuum model (PCM) using the integral equation formalism variant (IEFPCM) provided in the Gaussian 09 package. The vibrational spectra were simulated by convoluting the harmonic frequencies obtained at the same level of theory by a Lorentzian line shape (FWHM $4 \mathrm{~cm}^{-1}$ ). The calculated frequencies were scaled by 0.98 . All calculations were performed with the Gaussian 09 package. $^{15}$

\section{Molecular dynamics approaches}

To explicitly take into account the solvent disorder and temperature effect, we employed our recently introduced method combining First-Principle Molecular Dynamics (FPMD) simulations and the Nuclear Velocity Perturbation Theory (NVPT). ${ }^{32-34}$ FPMD simulations were used to sample the solute-solvent configurations and the generated trajectories were analysed in the NVPT framework to generate the VCD spectra.

FPMD simulations were performed using the CP2K package ${ }^{44}$ with the BLYP functional. ${ }^{45,}{ }^{46} \mathrm{We}$ employed the TZV2P-MOLOPT-GTH Gaussian basis set $^{47}$ and GTH pseudo potentials ${ }^{48-50}$ with a 0.5 fs time step, a density energy cut off of $400 \mathrm{Ry}$, and D3 dispersion correction. ${ }^{42,}{ }^{43}$ Simulations were performed on one isolated (S)-1-indanol molecule as well as one (S)-1-indanol molecule solvated in a periodic cubic box of 51 DMSO molecules.

For (S)-1-indanol solvated in DMSO, the box length was $18.38 \AA$. We first performed a 30 ps long trajectory in the canonical ensemble, at constant volume and temperature (NVT). The temperature was set to $\mathrm{T}=340 \mathrm{~K}$, slightly higher than the experimental temperature, in order to accelerate the dynamics as is usual in FPMD simulations. This NVT simulation was used to generate an equilibrium distribution of configurations. From this trajectory, we extracted 6 initial configurations (at times $t=10$, $14,18,22,26$ and $30 \mathrm{ps}$ ) from which we ran independent $20 \mathrm{ps}$ long trajectories in the microcanonical ensemble at constant volume and energy (NVE), to avoid perturbation of the dynamics by the thermostat. The 6 NVE trajectories were then analysed using NVPT.

For the isolated system, the box length was $14 \AA$. Thanks to the lower cost of this simulation relative to that in DMSO, the performed NVT trajectory was 170 ps long. After 10 ps of equilibration, we extracted from this trajectory 40 initial conformations, distant by $4 \mathrm{ps}$, to generate independent $20 \mathrm{ps}$ long NVE trajectories. The 40 NVE trajectories were then analysed using NVPT.

Calculations for determining the magnetic moment were performed on the NVE trajectories with our implementation of NVPT in the CPMD 4.1 package using a plane-wave basis set. ${ }^{34,51}$ For these calculations, the wavefunction energy cut off was set to $70 \mathrm{Ry}$ and we employed Trouiller-Martins pseudopotentials. ${ }^{52}$ The projected NVPT calculation of the electronic response properties was carried out every 4 fs of the microcanonical (NVE) trajectories. The electronic contributions were computed in the Wannier gauge from which we extracted molecular magnetic moments. ${ }^{34}$ For computing the 
VCD spectrum, we included all solvent molecules within a cutoff of $R_{0}=4 \square$ from the centre of mass of the (S)-1-indanol molecule, using the gauge invariant expression of the VCD signal, ${ }^{34}$ which includes a gauge transport term correcting the molecular magnetic moment. A damping function was used to select the solvent molecules to avoid sudden changes of the magnetic moment. This damping function was a Fermi distribution of width $D=0.25 \square$. All spectra shown have been smoothened in the frequency domain via convolution with a Gaussian filter with $\sigma=4 \mathrm{~cm}^{-1}$ in $\mathrm{CCl}_{4}$ and $6 \mathrm{~cm}^{-1}$ in DMSO and shifted down in frequency by $25 \mathrm{~cm}^{-1}$ for better comparison with the experiment.

Furthermore, the 30 ps NVT FPMD simulation of (S)-1-Indanol in DMSO was used as a way of generating clusters, i.e. as a conformational sampling tool. To this end, 124 snapshots were extracted from the NVT FPMD trajectory. For each snapshot a sphere was cut around the solute, with a cut-off of $4 \AA$. Using this radius, the sphere contains a 1:1 complex for all the obtained snap-shots. Each extracted snapshot was then optimized at the B3LYP/6-31G++(d,p) level, and the spectra were reconstructed as the sum of the 124 individual contributions.

\section{Results and discussion}

\section{III-A. Isolated molecule in the gas phase}

The different conformations of (S)-1-indanol are defined by two angles, shown in Scheme 1. The $\mathrm{C}_{9} \mathrm{C}_{4} \mathrm{C}_{3} \mathrm{C}_{2}\left(\tau_{1}\right)$ angle gives the pseudo-axial or pseudo-equatorial position of the hydroxyl group. The $\mathrm{OH}$ is in equatorial position for a positive $\tau_{1}$ angle, while it is in axial position when $\tau_{1}$ is negative. The $\mathrm{C}_{9} \mathrm{C}_{1} \mathrm{OH}\left(\tau_{2}\right)$ angle defines the orientation of the hydroxyl group relative to the aromatic ring of the molecule. A subscript $\mathbf{1}$ denotes a conformer with the hydroxyl pointing toward the aromatic ring, with $\mathrm{C}_{9} \mathrm{C}_{1} \mathrm{OH} \sim 60^{\circ}$. A subscript 2 corresponds to the hydroxyl pointing slightly outwards the ring, with $\mathrm{C}_{9} \mathrm{C}_{1} \mathrm{OH} \sim-60^{\circ}$. Finally, 3 denotes a hydroxyl group pointing completely outwards the molecule, with $\mathrm{C}_{9} \mathrm{C}_{1} \mathrm{OH} \sim-180^{\circ}$.

Two conformers have been clearly identified under jet-cooled conditions, namely $\mathbf{1 e q}$ and $\mathbf{2 a x} .^{36}$ The ratio between the two of them strongly depends on the cooling conditions. ${ }^{37,53}$ Two axial conformers 1ax and 2ax have been calculated with almost the same energy, but the barrier between them is small. So only one of them, 2ax, has been taken into account. A third isomer was experimentally identified in the laser-induced fluorescence spectrum and tentatively assigned to $\mathbf{3 e q}$.

\section{III-B. Non polar solvent}

\section{a. Static approach}

\section{IR spectrum}

The energy ordering of the different conformers calculated in a $\mathrm{CCl}_{4}$ continuum, as given by their relative Gibbs free energy at $300 \mathrm{~K}$, is slightly different from the gas phase results, for which the energies are calculated at $0 \mathrm{~K}$. The Gibbs free energies are listed in Table 1, together with the 
populations given by a Boltzmann distribution at room temperature. The four most stable conformers are the same 1eq, 1ax, 2ax and 3eq as calculated in the gas phase. The main difference is that $\mathbf{1 a x}$ is lower in energy than $\mathbf{2 a x}$ in $\mathrm{CCl}_{4}$ solution. Moreover, the population of $\mathbf{2 e q}$ and $\mathbf{3 a x}$, which was negligible in the gas phase, now amounts to $10 \%$ and $7 \%$, respectively. The most stable calculated structures are shown in Figure 1.

The simulated IR spectrum of (S)-1-indanol in a continuum of $\mathrm{CCl}_{4}$ is obtained by weighting the IR spectrum of each conformer by its Bolztmann population. It is shown in Figure 2, together with the experimental spectrum. The IR spectra of the individual conformations are shown in Figure S2 in ESI†.

Two vibration modes strongly contribute to the calculated spectrum: the $\beta(\mathrm{OH})$ bend, coupled with the asymmetric carbon $\beta(\mathrm{CH})$, is calculated in the $1380-1440 \mathrm{~cm}^{-1}$ range. It is strongly sensitive to the conformation of the molecule. While it appears with strong intensity in 1eq and 1ax, in which the $\mathrm{OH}$ group is directed inwards, it is much weaker in $\mathbf{2 a x}$ or $\mathbf{3 e q}$ in which the $\mathrm{OH}$ is directed outwards. The intensity of the $v(\mathrm{CO})$ calculated at $\sim 1060 \mathrm{~cm}^{-1}$ is also much stronger in 1eq and 1ax than in 2ax and 3eq. The doublet at $\sim 1500 \mathrm{~cm}^{-1}$ corresponds to modes involving mostly $\mathrm{C}_{\text {arom }} \mathrm{H}$ in-plane bending motions. The other peaks calculated in the spectrum are due to $\beta(\mathrm{CH})$ or $\beta\left(\mathrm{CH}_{2}\right)$ and cannot be described easily in terms of specific motions because of extensive delocalization.

The simulation obtained from the weighted spectra calculated in the harmonic approximation satisfactorily compares to the experiment, at least in terms of band positions. The $\beta(\mathrm{OH})$ band has much larger intensity in the calculated spectrum than in the experimental one. Figure S3 in ESI $\dagger$ displays the IR spectra of the individual conformations calculated at the B3LYP-D3 level. Comparison with Figure S2 shows that inclusion of dispersion does not modify the spectrum for a given geometry; it does not strongly modify the relative contributions of the different conformers to the total absorption spectrum.

\section{VCD spectrum}

The VCD spectrum of (S)-1-indanol in a $\mathrm{CCl}_{4}$ solution is shown in Figure 3a and the spectrum calculated from the weighted contributions of the six most stable conformers in Figure $3 b$. The VCD spectrum of each calculated conformer is given in Figure S4 in the ESI†, which shows that the intensity and even the sign of the bands strongly depend on conformation. A mild agreement only is obtained between the spectrum calculated in the frame of the double harmonic approximation and the experimental spectrum. While the position and sign of the bands is qualitatively satisfactory, discrepancies appear in the intensities, as it was already the case for the IR absorption spectrum. The relative intensity of the two components of the doublet at $1200 \mathrm{~cm}^{-1}$ is not well reproduced in the calculation. Moreover, the intensity ratio between the $\beta(\mathrm{OH})$ band at $\sim 1400 \mathrm{~cm}^{-1}$ and the doublet at $1200 \mathrm{~cm}^{-1}$ is by far overestimated, as it was in the IR spectrum. These bands correspond to modes involving the bending motions of the $\mathrm{OH}$ and of the aliphatic $\mathrm{CH}$. As already mentioned for the IR absorption spectrum, inclusion of dispersion does not substantially modify the VCD spectrum (Figure 
S5 in the ESI $\dagger$ ). A first explanation for that discrepancy could be that the energy ordering between the conformers is not correct. However, modification of the relative contributions of the conformers does not improve the spectrum. The disagreement is therefore not due to bad energy ordering but to the fact that optimised geometries are not representative of the large ensemble of conformations explored at room temperature. Thus, the agreement between experiment and simulations should be improved by resorting to FPMD calculations as described in what follows.

\section{b. Molecular-dynamics based spectrum}

\section{IR spectrum}

The IR spectrum obtained from the analysis of the FPMD trajectory in vacuum is shown in Figure 2c. It compares satisfactorily with the experiment in terms of band position. In terms of intensity, the 1400 $\mathrm{cm}^{-1}$ band assigned to $\beta(\mathrm{OH})$ is less intense than in the spectrum constructed from static calculations and shows better agreement with the experiment. However, the agreement between experiment and FPMD simulations is not perfect in the $v(\mathrm{CO})$ region. The use of BLYP could explain this discrepancy. Therefore, the six most stable conformers resulting from the static calculations described above have been recalculated at the BLYP level, using the same basis set. The energy ordering is slightly modified (see Table S1 in the ESI $\dagger$ ); in particular the contribution of 2 eq notably increases and that of 2ax decreases. The calculated spectra thus undergo some differences, as shown in Figure S6 in the ESI $\uparrow$. The intensity of the $\beta(\mathrm{OH})$ is still overestimated, but less than in the B3LYP simulations. The region of the $v(\mathrm{CO})$ in the $1000-1100 \mathrm{~cm}^{-1}$ range appears like a doublet in the BLYP spectrum because the $v(\mathrm{CO})$ stretch frequency is more conformer sensitive when BLYP is used. This band appears intense and red-shifted in the 2eq conformer, which explains the calculated doublet. These two effects explain the discrepancy between experimental and simulated spectra in the 1000$1100 \mathrm{~cm}^{-1}$ region.

\section{VCD spectrum}

The VCD spectrum obtained from the analysis of the FPMD trajectory in vacuum is shown in Figure 3c. It shows good agreement in terms of band position with the experiment shown in Figure $3 \mathrm{a}$. Moreover, the doublet at $\sim 1200 \mathrm{~cm}^{-1}$ is well reproduced in terms of relative intensity. The intensity ratio between the $\beta(\mathrm{OH})$ bend and the doublet at $\sim 1200 \mathrm{~cm}^{-1}$ is much closer to the experiment than that obtained from static calculations. In order to check the effect of the functional, the VCD spectra of the six most stable conformers of (S)-1-indanol in a $\mathrm{CCl}_{4}$ continuum have been calculated at the BLYP level and are shown in Figure S7 in the ESI $\dagger$. Overall, no strong difference in the agreement between experiment and static VCD spectrum is observed. As mentioned for the IR absorption, the better agreement obtained with FPMD simulations points to the fact that the optimised static geometries are not representative of the large ensemble of conformations explored at room temperature. 
Static methods are therefore satisfactory for IR, for which the position of the bands does not depend strongly on the conformation. For VCD, static methods give satisfactory band positions, but are less reliable for accounting for intensities, for which FPMD performs better, except in the region of 1440 $\mathrm{cm}^{-1}$ which is delicate to reproduce whatever the method used. Indeed, this region corresponds to a mode described in terms of $\beta(\mathrm{OH})$ bend, coupled with $\beta(\mathrm{CH})$ bends. The intensity of this mode, as well as its description in terms of atom displacements strongly depends on the conformation and the method used. It should be noted that although the FPMD simulations have been performed in vacuum, there is good agreement between simulations and experiments.

\section{III-C. DMSO solvent}

\section{a. Static approach}

\section{IR spectrum}

The experimental IR spectrum of (S)-1-indanol in DMSO is shown in Figure 4a. Only the region above $1100 \mathrm{~cm}^{-1}$ is shown, due to strong absorption of the solvent below this value. The main difference relative to $\mathrm{CCl}_{4}$ is the modification of the $1300-1500 \mathrm{~cm}^{-1}$ region. An intense narrow band is observed at $1325 \mathrm{~cm}^{-1}$ in $\mathrm{CCl}_{4}$ while a broad feature appears between 1400 and $1450 \mathrm{~cm}^{-1}$ in DMSO. This spectral change is by no means reproduced by the spectrum simulated using the weighted contributions of the most stable isolated monomers (population $>5 \%$ ) in a DMSO continuum, shown in Figure 4b). Indeed, the calculated spectrum of the (S)-1-indanol monomers exhibits an intense band at $1385 \mathrm{~cm}^{-1}$ which does not appear in the experimental spectrum. On the other hand, the strong feature experimentally observed at $1325 \mathrm{~cm}^{-1}$ is missing in the simulated spectrum. The strong disagreement between simulation and experiment indicates specific interactions between (S)-1-indanol and DMSO. This is not surprising in view of the hydrogen-bond accepting ability of DMSO. Indeed, Graton and co-workers have defined an elegant hydrogen-bond basicity scale based on IR spectroscopy, in which the hydrogen-bond donating strength of DMSO is classified as strong. ${ }^{54}$ According to this scale, all the molecules of an aliphatic alcohol like (S)-1-indanol are expected to be hydrogen bonded to the DMSO solvent. It seems therefore justified to consider a "cluster-in-a-bulk" approach starting from the 1:1 complex. The most stable calculated 1:1 complexes of (S)-1-indanol with DMSO-d6 in a DMSO continuum are shown in Figure 5. The IR absorption spectrum resulting from their weighted contribution is shown in Figure 4c. Their individual contributions are shown in Figure S8 in the ESI $\uparrow$. The energetic data calculated for the monomer and the 1:1 complexes are given in Table 2. It should be stressed here that the complexes used in the calculations result from a complete exploration of the PES, and are not designed following chemical intuition. The (S)-1-indanol geometries within the complex are identical to those calculated in $\mathrm{CCl}_{4}$. The four most stable complexes contain (S)-1-indanol in its equatorial form. This is reminiscent of what has been observed for jet-cooled (S)-1-indanol hydrates. ${ }^{37}$ Among them, the only one containing the most stable equatorial form (1eq) is the less stable. This geometry allows the DMSO to interact with the aromatic 
ring. In the other 1:1 structures, which contain (S)-1-indanol in 2eq or 3eq geometry, the OH group is pointing outwards and so does the DMSO. This indicates that structures with DMSO outside the aromatic ring are favoured.

The agreement between the spectrum simulated from the 1:1 complexes and the experiment is quite good in terms of position for the broad intense feature between 1250 and $1340 \mathrm{~cm}^{-1}$, assigned to the superposition of aliphatic $\mathrm{CH}$ bending modes calculated at 1320,1298 , and $1258 \mathrm{~cm}^{-1}$. The two sharp experimental bands at 1477 and $1460 \mathrm{~cm}^{-1}$ are also well reproduced by the simulated spectrum and are assigned to $\mathrm{CH}$ bending motions of different 1:1 complexes. However, the intensity of the $1325 \mathrm{~cm}^{-1}$ band is overestimated in the calculations. The only complex with bands of very weak intensity around $1325 \mathrm{~cm}^{-1}$ is that containing 1eq. This band is due to the $\mathrm{OH}$ bend coupled with the $\mathrm{C}^{*} \mathrm{H}$ bend localised on the chiral carbon. It is shifted down in frequency relative to the bare molecule in all the complexes except in that with 1eq, in which this mode is calculated at $1415 \mathrm{~cm}^{-1}$.

It seems relevant to focus on the 1:1 complexes because (S)-1-indanol has only one hydrogen bonding site. However, the 1:2 complexes were also calculated, using the same calculation strategy. The IR absorption spectrum obtained from the weighted contribution of the most stable 1:2 complexes in a DMSO continuum is displayed in Figure $4 \mathrm{~d}$. The agreement with experiment is satisfactory for the $1: 2$ complexes as well, at least in terms of band positions. More discrepancies are observed for the band intensities than for the 1:1 complex. This indicates that taking into account the first hydrogen-bonded solvent molecule is necessary for satisfactorily simulating the spectrum. However, introducing a second solvent molecule does not strongly perturb the system, as seen in the calculated IR absorption spectra of the 1:1 and 1:2 complexes.

\section{VCD spectrum}

Figure 6 displays the experimental VCD spectrum together with that simulated from the weighted contributions of the most stable (S)-1-indanol conformers in a DMSO continuum, as well as that obtained from the most stable (S)-1-indanol:DMSO-d6 and (S)-1-indanol-(DMSO-d6) 2 complexes. The individual contributions of the 1:1 complexes used for the weighted average are shown in Figure S9 in the ESI $\dagger$. The agreement between the experiment and the spectrum of the 1:1 complex is overall satisfactory. The main discrepancy appears in the $1200 \mathrm{~cm}^{-1}$ region, where the experimental spectrum displays two intense features while only one is calculated. Moreover, the intensity of the $\beta(\mathrm{OH})$ bend is overestimated, as it was in the IR absorption or in the VCD and IR spectra in $\mathrm{CCl}_{4}$. The second lowenergy conformer (S)-1-Indanol:DMSO-d6 3eq exhibits a doublet near $1200 \mathrm{~cm}^{-1}$, as the experimental spectrum does, while the three other complexes based on 1eq or $\mathbf{2 e q}$ exhibit only one significant band. 3eq is also the conformer that reproduces well the region around $1475 \mathrm{~cm}^{-1}$. We can suppose that the contribution of this conformer is underestimated by static calculations. Increasing the contribution of 3eq would also decrease the intensity of the band at $1413 \mathrm{~cm}^{-1}$, which is overestimated in the calculated averaged spectrum and arises only from the three other complexes. The same simulation has been performed at the B3LYP-D3/6-31++G(d,p) level of theory (see Figure S10 and Table S2 in 
ESI $\dagger$ ). The agreement with experiment is less satisfactory. The main difference with the B3LYP/6$31++\mathrm{G}(\mathrm{d}, \mathrm{p})$ calculations is that the interaction between DMSO and the aromatic ring is favoured when dispersion is included. As the result, the most stable calculated complex contains 1eq, and three out of the four most stable complexes contain 1ax, which strongly modifies the spectrum. Comparison between experimental and calculated spectra shows that the contribution of the complexes with DMSO interacting with the aromatic ring is overestimated when D3 is included, as it has been suggested already by Bunnemann and Merten in their VCD study of a carboxylic acid in DMSO by a "cluster-in-a-bulk" approach. ${ }^{55}$ Even when the (S)-1-indanol keeps the same geometry, including dispersion results to a modification of the DMSO position hence a change in the hydrogen bond geometry. This has been shown to strongly influence the VCD signal of the modes localised on the bound $\mathrm{OH}^{56}$ In what follows, we shall therefore limit the discussion to the B3LYP/6-31++G(d,p) calculations.

The spectrum simulated from the weighted contribution of the most stable (S)-1-indanol:(DMSO-d6) complexes also shows satisfactory agreement with the experiment. As observed for the IR absorption, the first solvent molecule is mandatory for accounting for the solvent effect on the VCD spectrum, but the presence of the second one does not strongly perturb the system. We could use a sum of 1:1 and 1:2 clusters to get better agreement between experimental and calculated spectrum. This approach has been successfully proposed by $\mathrm{Xu}$ and co-workers. ${ }^{25}$ The relative weight of $1: 1$ and 1:2 complexes does not rest on a thermodynamics-based distribution of cluster abundance but is adjusted to get better agreement between experimental and calculated spectrum. However, imperfect agreement in terms of intensity was already observed in the non-interacting $\mathrm{CCl}_{4}$ solvent; this problem would not be solved by considering larger-size clusters. Finally, (S)-1-indanol has only one hydrogen bonding site. The first molecule is thus strongly bound, in contrast to the second molecule whose location is more sensitive to the method hence more difficult to determine. ${ }^{55}$ For these different reasons, we have not chosen to include the 1:2 complexes. However, we conclude from the static calculations at the B3LYP/6-31G++(d,p) level that the "cluster-in-bulk" approach gives satisfactory results when the 1:1 complexes are considered, but that the presence of 1:2 complexes cannot be ruled out. The results of the FPMD simulations will now be discussed in the light of these observations.

\section{b. Conformational sampling from NVT simulation}

The VCD spectrum obtained as an average over 124 structures extracted from the NVT FPMD trajectory (see methods) is shown in Figure 7c. It should be noted that the sphere defined by the cutoff contains a 1:1 complex for all the obtained snapshots, which is in line with both hydrogen-bond scales and cluster-in-the-bulk findings that the first solvent molecule has the major effect on the shape of the VCD spectrum. Optimization of the snapshots mostly leads to equatorial conformers $(41 \%$ and $30 \%$ for $\mathbf{3 e q}$ and $\mathbf{2 e q}$, respectively). The contribution of $\mathbf{3 e q}$ is larger here than in static calculations (41\% vs. 34\%). A stronger contribution of this conformer, which has no strong VCD signal in the 1250 and $1340 \mathrm{~cm}^{-1}$ range, results to better calculated intensity of the $1325 \mathrm{~cm}^{-1}$ feature. The $3 \mathbf{a x}$ and 
2ax conformers represent 16 and $13 \%$ of the snapshots, respectively. This contrasts with the static calculations, in which all the 1:1 complexes are of equatorial nature. FPMD calculations allow thus exploring axial conformations of (S)-1-indanol, especially at the beginning of the trajectory as shown in Figure 8. The spectrum of $3 \mathbf{a x}$ shows a band at $1271 \mathrm{~cm}^{-1}$. The experimental spectrum shows a positive band in this region, which is not well reproduced by the cluster-in-a-bulk approach. The intensity of the $1425 \mathrm{~cm}^{-1}$ band $(\beta(\mathrm{OH})$ bend) is more satisfactory than in static calculations, as is that of the doublet at $\sim 1200 \mathrm{~cm}^{-1}$. Improvement of the spectrum is therefore obtained because higherenergy regions of the PES, explored by the system at room temperature, are better sampled by FPMD simulations then by conisdering only the most stable conformers.

Introduction of dispersion in the optimization of the snapshots does not modify the statistics in terms of (S)-1-indanol conformations.

\section{c. Analysis of NVE MD trajectories.}

As described before, we first ran six independent NVE trajectories starting from six initial configurations (at times $\mathrm{t}=10,14,18,22,26$ and $30 \mathrm{ps}$ ) extracted from the NVT trajectory. These trajectories were first used to simulate the IR spectrum. Because of the trajectories are limited to $20 \mathrm{ps,}$ full sampling of the PES is not expected. We therefore used the correspondence between simulated and experimental IR spectra for selecting the reliable NVE trajectories. The spectra simulated from the six trajectories yield similar results in the $1600-1250 \mathrm{~cm}^{-1}$ range, and satisfactorily reproduce the experimentally observed spectral patterns in this region. Differences between trajectories are however observed in the $1200 \mathrm{~cm}^{-1}$ range, which is crucial because it is difficult to reproduce in the VCD spectrum. It turns out the trajectories at 18 and $22 \mathrm{ps}$ give the best IR spectrum in the $1200 \mathrm{~cm}^{-1}$ range (doublet at 1180-1220 $\mathrm{cm}^{-1}$ ). In what follows we will discuss the IR and VCD spectra obtained from the averaged spectra resulting from the analysis of these two NVE FPMD trajectories.

\section{IR spectrum}

The IR spectrum resulting from the analysis of the two selected NVE FPMD trajectories is shown in Figure 9. Notwithstanding the broadening of the bands in the experimental spectrum, it shows good agreement with the experiment. The relative intensities are better reproduced than in static calculations in particular for the $1325 \mathrm{~cm}^{-1}$ band, the intensity of which was overestimated in the static spectra.

\section{VCD spectrum}

The VCD spectrum simulated from the analysis of the NVE MD trajectory is shown in Figure 7d. The agreement with the experiment is satisfactory. A doublet clearly appears at $1200 \mathrm{~cm}^{-1}$, with however slightly weaker intensity than expected. The region below $1350 \mathrm{~cm}^{-1}$, as well as that above $1450 \mathrm{~cm}^{-1}$, is well reproduced by the simulation. A strong discrepancy is observed at $\sim 1415 \mathrm{~cm}^{-1}$, where a calculated positive peak does not find its counterpart in the experiment. The intensity of the band at $1457 \mathrm{~cm}^{-1}$ is also overestimated in the calculations. This corresponds to the region of the $\mathrm{CH}_{2}$ scissoring and the $\beta(\mathrm{OH})$ bending modes. This region is especially sensitive to the functional used. The intense band in the $1320 \mathrm{~cm}^{-1}$ range $\left(\beta(\mathrm{OH})\right.$ mixed with $\mathrm{C}^{*} \mathrm{H}$ bending motions) is more intense 
and of lower-frequency at the B3LYP than at the BLYP level. Moreover, the description of the modes is not identical with the two functionals, and modes involving the $\mathrm{CH}$ bends mixed with the $\mathrm{OH}$ bend are also modified, which may explain the observed discrepancy.

\section{d. Trajectory analysis - Comparison with the "cluster-in-the-bulk" method}

Analysis of the NVT trajectory indicates that the solute is most of the time bound to one DMSO molecule, and only one (Figure 10a and c). The hydrogen bond is strong enough not to be disrupted more than a few times during the 20 ps trajectory, so that another H-bond with another solvent molecule is formed. This confirms the preferential formation of stable 1:1 complexes and justifies the use of the "cluster-in-the-bulk" model. The (S)-1-indanol is sometimes involved in a bifurcated hydrogen bond with two molecules of DMSO (Figure 10b); this geometry corresponds to the transition state between two differently-oriented 1:1 complexes. The hydrogen bond between the first DMSO (Figure 10a) is broken so that a new hydrogen bond can form with the second DMSO molecule (Figure 10c). This transition state can be seen as a 1:2 complex, which is not in an equilibrium geometry. This could justify a posteriori the inclusion of carefully-designed 1:2 complexes in the cluster-in-the-bulk simulation of the spectrum, although these 1:2 structures are not long-lived complexes. $^{25}$

The question can be raised whether the exploration of the conformational space of (S)-1-indanol is complete during a NVE trajectory. Comparison between the two NVE trajectories used for simulating the IR absorption and VCD spectra (started at 18 and 22 ps of the NVT trajectory) can be obtained by analysing the histogram of $\tau_{1}$ and $\tau_{2}$ angles shown in Figure 11. The detailed evolution of $\tau_{1}$ and $\tau_{2}$ with time is shown in Figure S11 in the ESI $\dagger$. In the trajectory starting at 22 ps, $\tau_{1}$ clearly shows a bimodal distribution with maxima at $\sim-15$ and $+20^{\circ}$, corresponding to axial and equatorial positions, with however predominant equatorial position. $\tau_{2}$ also shows a bimodal distribution at $\sim-80^{\circ}$ and $\sim-$ $150^{\circ}$, which corresponds to the $\mathrm{OH}$ directed slightly $\left(-80^{\circ}\right)$ or completely $\left(-150^{\circ}\right)$ outwards the molecule. This geometry favours formation of the hydrogen bond to the solvent. The most frequent structure, appearing as a red spot in Figure 11, corresponds to the most stable 2eq complex obtained in static calculations. The other maximum is of axial nature, and is not obtained as a stable structure in static calculations. It corresponds to 3ax, which is slightly populated in the bare molecule (see Table 1).

In the trajectory starting at $18 \mathrm{ps}$, the $\tau_{1}$ distribution shows one broad maximum only, encompassing axial and equatorial positions. The maximum at $\tau_{2} \sim-150^{\circ}$ disappears, but a weak maximum at $\sim 50^{\circ}$, which corresponds to $\mathrm{OH}$ directed inwards, is observed. The most abundant geometry corresponds to 2ax of the bare monomer, although 2eq, like in the trajectory starting at $22 \mathrm{ps}$ and in the static calculations, also strongly contributes to the spectrum.

Both trajectories therefore give the most stable 2eq complex obtained in static calculations as the most abundant or one of the most abundant species. However, differences between the two NVE trajectories analysed here indicate that the sampling of the PES is not complete during the trajectory, in contrast 
with the trajectories in vacuum, due to the long hydrogen bond lifetime. Therefore, the relative contributions of the different structures can be deduced from these trajectories in a reliable way only if the initial conditions are carefully chosen. Longer trajectories would be highly desirable but computationally less demanding methods should be used. In this respect, polarisable force fields provide an interesting alternative for calculating the VCD spectra of complex molecules in realistic environments. ${ }^{57,58}$

\section{Conclusions}

This study allows assessing the role of flexibility and hydrogen bond formation on the IR absorption and VCD spectrum of a medium-size floppy molecule. (S)-1-indanol displays a low-frequency puckering motion as well as several rotamers due to the $\mathrm{OH}$ position. In an inert solvent like $\mathrm{CCl}_{4}$, static calculations yield the same most stable conformers as in the gas phase. The absorption and VCD spectra are satisfactorily accounted for by considering the solvent as a continuum. However, discrepancies in the intensities are observed. First principles molecular dynamics simulations (FPMD) resting on the nuclear velocity perturbation theory (NVPT) perform better in reproducing the relative intensities. This is because FPMD calculations include the large ensemble of conformations explored at room temperature. This allows including conformers that are neglected by static calculations at room temperature, or conformers that do not correspond to a minimum-energy structure.

Strong hydrogen bond is expected between (S)-1-indanol and the DMSO solvent. This is confirmed by both FPMD simulations and static calculations. Indeed, cluster-in-the-bulk calculations taking into account the most stable 1:1complexes give satisfactory results. The choice of the geometry of the clusters taken as an input of the static calculations is very important and the complexes used here result from a full exploration of the potential-energy surface. The sensitivity of the static calculations to the input geometries is due to the fact that, for a given conformation of (S)-1-indanol, the shape of the VCD spectrum, in particular for modes involving motions localized on $\mathrm{OH}$, is very sensitive to the position of DMSO, namely, the directionality of the hydrogen bond. Improvement of the agreement between theory and experiment is obtained when IR absorption and VCD spectra are calculated from the summed contributions of snapshots taken from a NVT MD trajectory and subsequently optimized at the B3LYP/6-31G++(d,p) level. The sampling of the PES is indeed better this way than when taking only the most stable forms because higher-energy regions of the potential-energy surface are explored. Moreover, the calculations include the effect of all the solvent molecules. IR absorption and VCD spectra are also calculated in the frame on NVPT theory from the analysis of shorter NVE trajectories that are chosen, among those run, on the basis of a good description of the IR absorption. Both static calculations and FPMD simulations show that one DMSO molecule interacts preferentially with the solute, which validates the cluster-in-the-bulk approach. Moreover, the most abundant structure found in the FPMD trajectories is close to the most stable cluster-in-the-bulk 1:1 complex. VCD is an 
original way of probing the interaction with the first solvent molecule, which is satisfactorily described by a cluster-in-the-bulk approach. On the other hand, FPMD simulations are unrivalled tools for describing systems with very anharmonic modes, like the puckering motion of 1-indanol, that explore high-energy regions of the potential-energy surface at room temperature. Therefore, it includes non-equilibrium structures, for example the intermediate state between two 1:1 solute:solvent complexes, a 1:2 structure which is not a local minimum.

\section{Acknowledgements}

We thank Anaël Danielou and Jérémy Lefebvre for experimental help. Support from the French National Research Agency (ANR) is acknowledged (Grant ANR-08-BLAN-0158), as well as computer time allowances by DI Univ. Paris-Sud. We also acknowledge the use of the computing facility cluster MésoLUM of the LUMAT federation (FR LUMAT 2764). The research described here has been supported by Triangle de la physique contract Dichromol no. 2007-025T.

\section{References}

1. L. A. Nafie, Annu. Rev. Phys. Chem., 1997, 48, 357-386.

2. P. L. Polavarapu and J. T. He, Analytical Chemistry, 2004, 76, 61A-67A.

3. G. Magyarfalvi, G. Tarczay and E. Vass, Wiley Interdisciplinary Reviews-Computational Molecular Science, 2011, 1, 403-425.

4. A. J. Hutt, Cns Spectrums, 2002, 7, 14-22.

5. T. B. Freedman, X. L. Cao, R. K. Dukor and L. A. Nafie, Chirality, 2003, 15, 743-758.

6. A. Bouchet, T. Brotin, D. Cavagnat and T. Buffeteau, Chemistry-a European Journal, 2010, 16, 4507-4518.

7. A. Sen, A. Bouchet, V. Lepere, K. Le Barbu-Debus, D. Scuderi, F. Piuzzi and A. Zehnacker-Rentien, The Journal of Physical Chemistry. A, 2012, 116, 8334-8344.

8. C. Merten, F. Li, K. Bravo-Rodriguez, E. Sanchez-Garcia, Y. Xu and W. Sander, Physical Chemistry Chemical Physics, 2014, 16, 5627-5633.

9. C. H. Pollok and C. Merten, Physical Chemistry Chemical Physics, 2016, 18, 1349613502. 
10. C. Merten, V. Smyrniotopoulos and D. Tasdemir, Chemical Communications, 2015, 51, 16217-16220.

11. T. A. Keiderling, Current Opinion in Chemical Biology, 2002, 6, 682-688.

12. L. Ducasse, F. Castet, A. Fritsch, I. Huc and T. Buffeteau, Journal of Physical Chemistry A, 2007, 111, 5092-5098.

13. J. R. Cheeseman, M. J. Frisch, F. J. Devlin and P. J. Stephens, Chem. Phys. Lett., 1996, 252, 211-220.

14. P. J. Stephens, Journal of Physical Chemistry, 1985, 89, 748-752.

15. M. J. Frisch, G. W. Trucks, H. B. Schlegel, G. E. Scuseria, M. A. Robb, J. R. Cheeseman, G. Scalmani, V. Barone, B. Mennucci, G. A. Petersson, H. Nakatsuji, M. Caricato, X. J. Li, H. P. Hratchian, A. F. Izmaylov, J. Bloino, G. Zheng, J. L. Sonnenberg, M. Hada, M. Ehara, K. Toyota, R. Fukuda, J. Hasegawa, M. Ishida, T. Nakajima, Y. Honda, O. Kitao, H. Nakai, T. Vreven, J. Montgomery, J. A. , J. E. Peralta, F. Ogliaro, M. Bearpark, J. J. Heyd, E. Brothers, K. N. Kudin, V. N. Staroverov, R. Kobayashi, J. Normand, K. Raghavachari, A. Rendell, J. C. Burant, S. S. Iyengar, J. Tomasi, M. Cossi, N. Rega, J. M. Millam, M. Klene, J. E. Knox, J. B. Cross, V. Bakken, C. Adamo, J. Jaramillo, R. Gomperts, R. E. Stratmann, O. Yazyev, A. J. Austin, R. Cammi, C. Pomelli, J. W. Ochterski, R. L. Martin, K. Morokuma, V. G. Zakrzewski, G. A. Voth, P. Salvador, J. J. Dannenberg, S. Dapprich, A. D. Daniels, O. Farkas, J. B. Foresman, J. V. Ortiz, J. Cioslowski and D. J. S. Fox, Gaussian Inc., Wallingford CT2009, pp. Gaussian 09, Revision A.02.

16. M. Heshmat, E. J. Baerends, P. L. Polavarapu and V. P. Nicu, Journal of Physical Chemistry A, 2014, 118, 4766-4777.

17. F. J. Devlin, P. J. Stephens and P. Besse, Journal of Organic Chemistry, 2005, 70, 2980-2993.

18. J. Bloino and V. Barone, Journal of Chemical Physics, 2012, 136, 124108.

19. V. Barone and C. Minichino, Theochem-Journal of Molecular Structure, 1995, 330, 365-376.

20. C. Merten, J. Bloino, V. Barone and Y. Xu, Journal of Physical Chemistry Letters, 2013, 4, 3424-3428.

21. J. Kessler, T. A. Keiderling and P. Bour, Journal of Physical Chemistry B, 2014, 118, 6937-6945. 
22. C. Merten and Y. Xu, Angewandte Chemie-International Edition, 2013, 52, 20732076.

23. J. Sadlej, J. C. Dobrowolski and J. E. Rode, Chem. Soc. Rev., 2010, 39, 1478-1488.

24. J. E. Rode, M. H. Jamroz, J. C. Dobrowolski and J. Sadlej, Journal of Physical Chemistry A, 2012, 116, 7916-7926.

25. M. Losada and Y. J. Xu, Physical Chemistry Chemical Physics, 2007, 9, 3127-3135.

26. G. Tarczay, S. Gobi, E. Vass and G. Magyarfalvi, Vibrational Spectroscopy, 2009, 50, 21-28.

27. E. Debie, L. Jaspers, P. Bultinck, W. Herrebout and B. Van der Veken, Chem. Phys. Lett., 2008, 450, 426-430.

28. V. P. Nicu, E. Debie, W. Herrebout, B. Van der Veken, P. Bultinck and E. J. Baerends, Chirality, 2009, 21, E287-E297.

29. K. J. Jalkanen, R. M. Nieminen, M. Knapp-Mohammady and S. Suhai, International Journal of Quantum Chemistry, 2003, 92, 239-259.

30. J. Kubelka, R. Huang and T. A. Keiderling, Journal of Physical Chemistry B, 2005, 109, 8231-8243.

31. A. S. Perera, J. Thomas, M. R. Poopari and Y. J. Xu, Frontiers in Chemistry, 2016, 4.

32. A. Scherrer, F. Agostini, D. Sebastiani, E. K. U. Gross and R. Vuilleumier, The Journal of chemical physics, 2015, 143, 074106-074106.

33. A. Scherrer, R. Vuilleumier and D. Sebastiani, The Journal of Chemical Physics, 2016, 145, 084101.

34. A. Scherrer, R. Vuilleumier and D. Sebastiani, Journal of Chemical Theory and Computation, 2013, 9, 5305-5312.

35. J. Kapitan, V. Baumruk and P. Bour, Journal of the American Chemical Society, 2006, 128, 2438-2443.

36. K. Le Barbu-Debus, F. Lahmani, A. Zehnacker-Rentien, N. Guchhait, S. S. Panja and T. Chakraborty, Journal of Chemical Physics, 2006, 125, 174305. 
37. A. Bouchet, J. Altnoder, M. Broquier and A. Zehnacker, Journal of Molecular Structure, 2014, 1076, 344-351.

38. J. Altnoeder, A. Bouchet, J. J. Lee, K. E. Otto, M. A. Suhm and A. ZehnackerRentien, Physical Chemistry Chemical Physics, 2013, 15, 10167-10180.

39. B. Velino, P. Ottaviani, W. Caminati, A. Giardini and A. Paladini, Chemphyschem, 2006, 7, 565-568.

40. MacroModel version 9.8; ed. Schrödinger, LLC: New York, NY, 2010, 2010.

41. M. D. Halls, J. Velkovski and H. B. Schlegel, Theoretical Chemistry Accounts, 2001, $\mathbf{1 0 5}, 413$.

42. S. Grimme, J. Antony, S. Ehrlich and H. Krieg, Journal of Chemical Physics, 2010, 132.

43. S. Grimme, S. Ehrlich and L. Goerigk, Journal of Computational Chemistry, 2011, 32, 1456-1465.

44. J. VandeVondele, M. Krack, F. Mohamed, M. Parrinello, T. Chassaing and J. Hutter, Computer Physics Communications, 2005, 167, 103-128.

45. A. D. Becke, Physical Review A, 1988, 38, 3098-3100.

46. C. Lee, W. Yang and R. G. Parr, Physical Review B, 1988, 37, 785.

47. J. VandeVondele and J. Hutter, Journal of Chemical Physics, 2007, 127.

48. S. Goedecker, M. Teter and J. Hutter, Physical Review B, 1996, 54, 1703-1710.

49. C. Hartwigsen, S. Goedecker and J. Hutter, Physical Review B, 1998, 58, 3641-3662.

50. M. Krack, Theoretical Chemistry Accounts, 2005, 114, 145-152.

51. CPMD, http://www.cpmd.org/

Copyright IBM Corp 1990-2015

Copyright MPI für Festkörperforschung Stuttgart 1997-2001.

52. N. Troullier and J. L. Martins, Physical Review B, 1991, 43, 1993-2006. 
53. D. Scuderi, A. Paladini, M. Satta, D. Catone, S. Piccirillo, M. Speranza and A. G. Guidoni, Physical Chemistry Chemical Physics, 2002, 4, 4999-5003.

54. C. Laurence, J. Graton, M. Berthelot, F. Besseau, J. Y. Le Questel, M. Lucon, C. Ouvrard, A. Planchat and E. Renault, Journal of Organic Chemistry, 2010, 75, 41054123.

55. K. Bunnemann and C. Merten, Physical Chemistry Chemical Physics, 2017.

56. V. P. Nicu, M. Heshmat and E. J. Baerends, Physical Chemistry Chemical Physics, 2011, 13, 8811-8825.

57. J. W. Ponder, C. J. Wu, P. Y. Ren, V. S. Pande, J. D. Chodera, M. J. Schnieders, I. Haque, D. L. Mobley, D. S. Lambrecht, R. A. DiStasio, M. Head-Gordon, G. N. I. Clark, M. E. Johnson and T. Head-Gordon, Journal of Physical Chemistry B, 2010, 114, 2549-2564.

58. F. Thaunay, J. P. Dognon, G. Ohanessian and C. Clavaguéra, Phys. Chem. Chem. Phys., 2015, 17, 25968-25977. 
Table 1 Energetic and structural parameters of the most stable conformers of (S)-1-indanol in $\mathrm{CCl}_{4}$ continuum calculated at the B3LYP/6-31G $++(d, p)$ level. The Boltzmann population is given at $300 \mathrm{~K}$.

\begin{tabular}{|l|r|r|r|r|}
\hline Geometry & $\begin{array}{c}\text { Gibbs free } \\
\text { energy } \\
(\mathrm{kcal} / \mathrm{mol})\end{array}$ & $\begin{array}{c}\text { Boltzmann } \\
\text { population } \\
(\%)\end{array}$ & $\begin{array}{r}\mathrm{C}_{9} \mathrm{C}_{4} \mathrm{C}_{3} \mathrm{C}_{2} \\
\text { Angle }\left(^{\circ}\right)\end{array}$ & $\begin{array}{c}\mathrm{C}_{9} \mathrm{C}_{1} \mathrm{OH} \\
\text { Angle }\left(^{\circ}\right)\end{array}$ \\
\hline 1eq & 0.00 & 33 & 16.8 & 55.1 \\
\hline $1 \mathrm{ax}$ & 0.30 & 20 & -13.2 & 43.0 \\
\hline $2 \mathrm{ax}$ & 0.35 & 18 & -14.7 & -52.0 \\
\hline $3 \mathrm{eq}$ & 0.58 & 12 & 16.4 & -168.0 \\
\hline 2eq & 0.70 & 10 & 16.0 & -76.0 \\
\hline $3 \mathrm{ax}$ & 0.94 & 7 & -15.3 & -176.5 \\
\hline
\end{tabular}

Table 2 Energetic parameters of the most stable (S)-1-indanol/DMSO 1:1 complexes in DMSO- $\mathrm{d}_{6}$ continuum calculated at the $\mathrm{B} 3 \mathrm{LYP} / 6-31 \mathrm{G}++(\mathrm{d}, \mathrm{p})$ level. The Boltzmann population is given at $300 \mathrm{~K}$.

\begin{tabular}{|l|r|r|r|r|}
\hline Geometry & $\begin{array}{c}\text { Gibbs free } \\
\text { energy } \\
(\mathrm{kcal} / \mathrm{mol})\end{array}$ & $\begin{array}{c}\text { Boltzmann } \\
\text { population } \\
(\%)\end{array}$ & $\begin{array}{c}\mathrm{C}_{9} \mathrm{C}_{4} \mathrm{C}_{3} \mathrm{C}_{2} \\
\text { Angle }\left(^{\circ}\right)\end{array}$ & $\begin{array}{c}\mathrm{C}_{9} \mathrm{C}_{1} \mathrm{OH} \\
\text { Angle }\left(^{\circ}\right)\end{array}$ \\
\hline 2eq-a & 0.00 & 40 & 16.6 & -78.5 \\
\hline 3eq & 0.10 & 34 & 16.8 & -163.0 \\
\hline 2eq-b & 0.43 & 20 & 16.7 & -85.2 \\
\hline 1eq & 1.12 & 6 & 16.6 & 57.9 \\
\hline
\end{tabular}




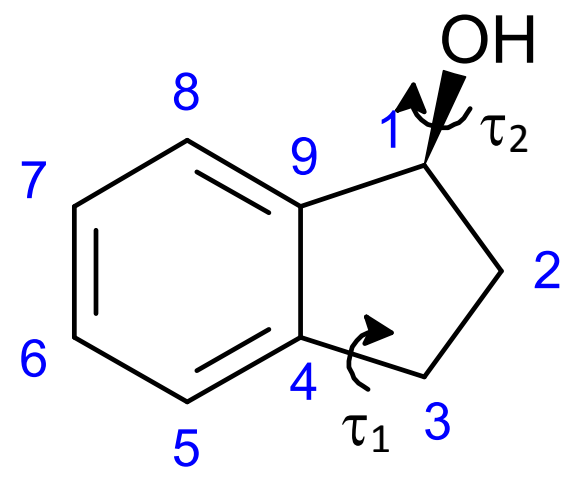

Scheme 1: Scheme of (S)-1-indanol and atom numbering 


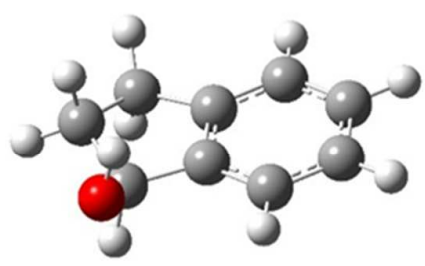

1eq : $33 \%$

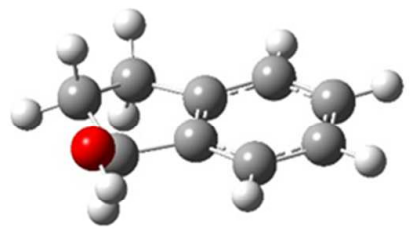

2eq : $10 \%$

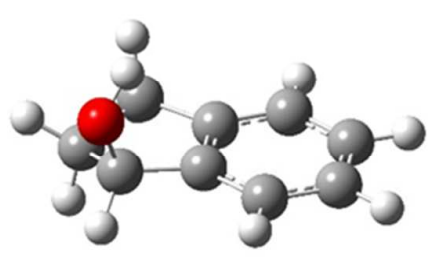

1ax : $20 \%$

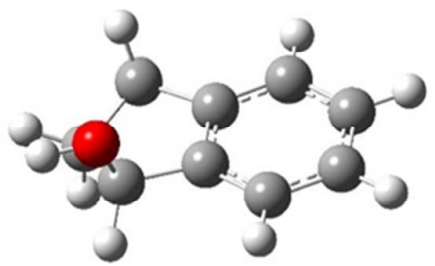

3ax : 7\%

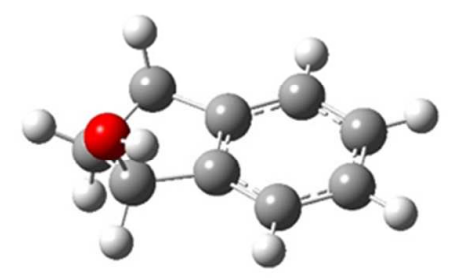

$2 \mathrm{ax}: 18 \%$

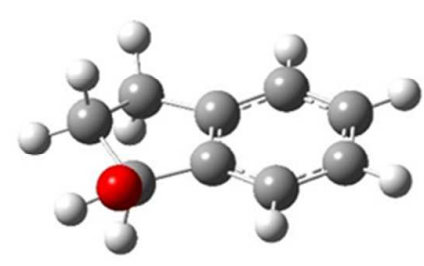

3eq : $12 \%$

Figure 1: Most stable structures of (S)-1-indanol in $\mathrm{CCl}_{4}$ continuum calculated at the $\mathrm{B} 3 \mathrm{LYP} / 6-31++\mathrm{g}(\mathrm{d}, \mathrm{p})$ level. The relative abundances are given in $\%$ of the total (S)-1-indanol population. 


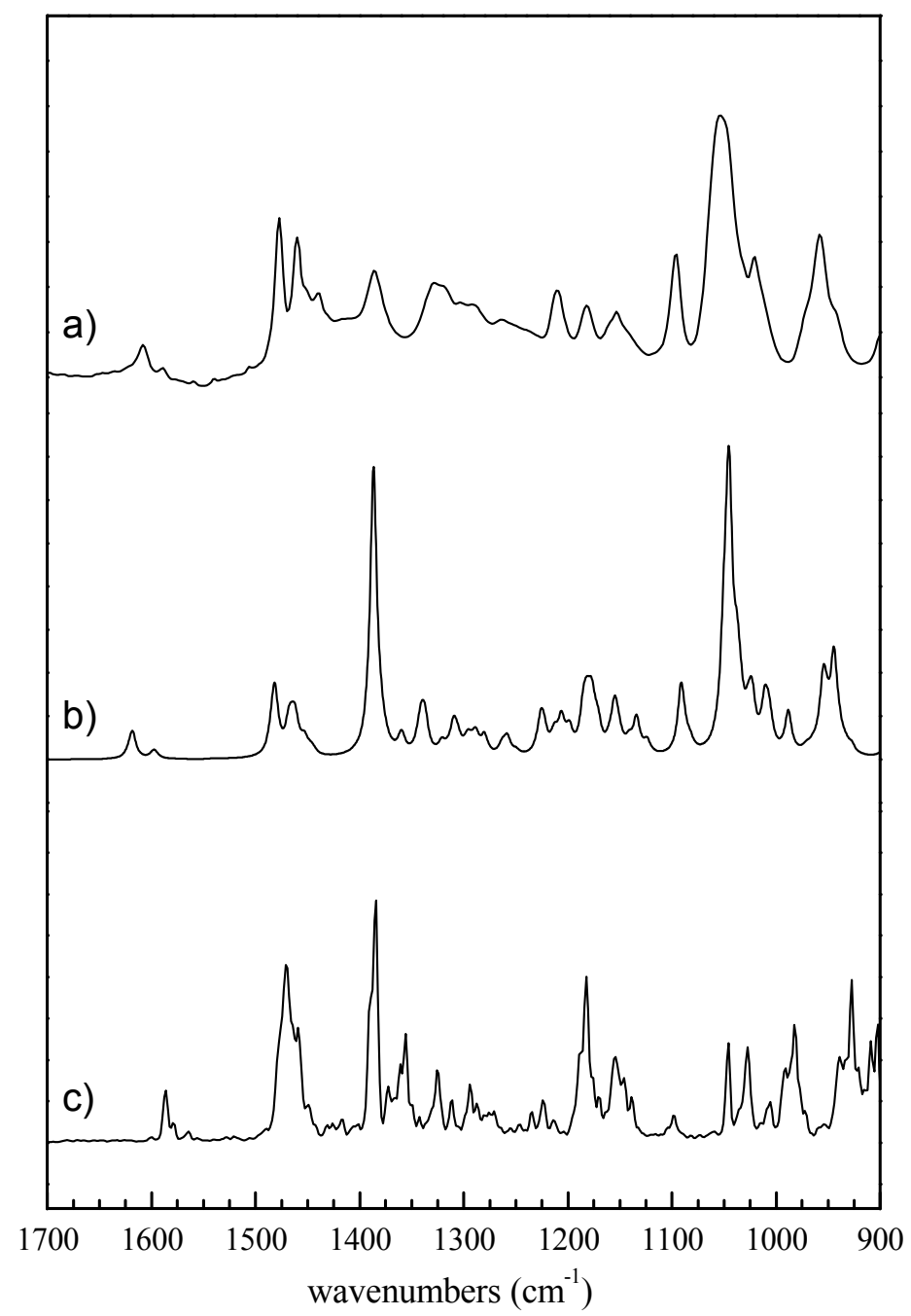

Figure 2: a) Experimental IR absorption spectrum of (S)-1-indanol in $\mathrm{CCl}_{4}$ b) Simulated IR absorption spectrum obtained from the weighted IR absorption spectra of the most stable (S)-1-indanol structures in a $\mathrm{CCl}_{4}$ continuum, calculated at the $\mathrm{B} 3 \mathrm{LYP} / 6$ $31 \mathrm{G}++(\mathrm{d}, \mathrm{p})$ level c) Simulated IR absorption spectrum obtained from the analysis of a NVE $\mathrm{ab}$ initio molecular dynamics trajectory in vacuum. The spectrum is convoluted by a Gaussian $\left(4 \mathrm{~cm}^{-1} \mathrm{FWMH}\right)$ and shifted down in energy by $25 \mathrm{~cm}^{-1}$. 


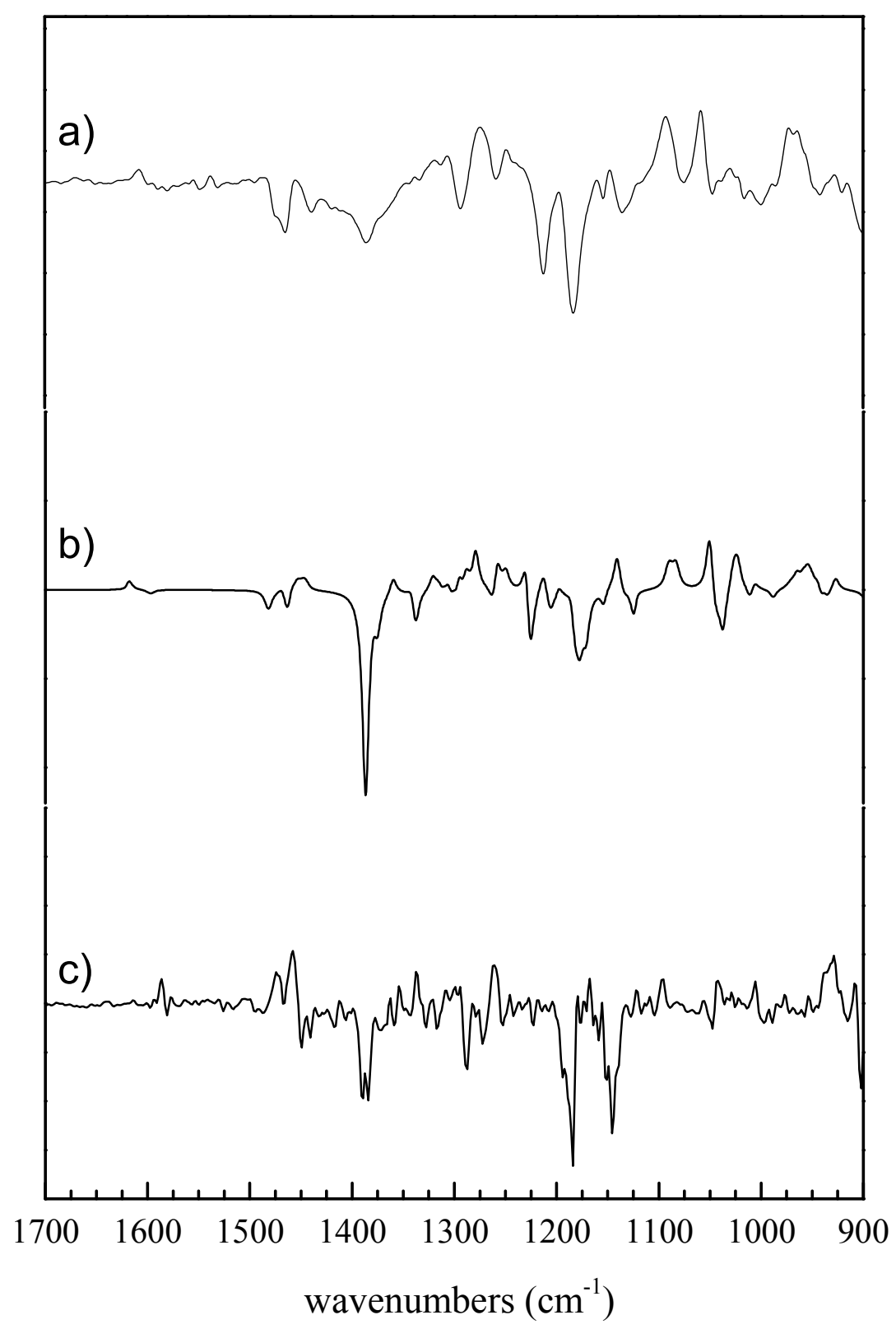

Figure 3: a) Experimental VCD spectrum of (S)-1-indanol in $\mathrm{CCl}_{4}$ b) Simulated VCD spectrum obtained from the weighted VCD spectra of the most stable (S)-1-indanol structures in a $\mathrm{CCl}_{4}$ continuum, calculated at the B3LYP/6-31G++(d, p) level c) Simulated VCD spectrum obtained from the analysis of a NVE ab initio molecular dynamics trajectory in vacuum. The spectrum is convoluted by a Gaussian $\left(4 \mathrm{~cm}^{-1} \mathrm{FWMH}\right)$ and shifted down in energy by $25 \mathrm{~cm}^{-1}$. 


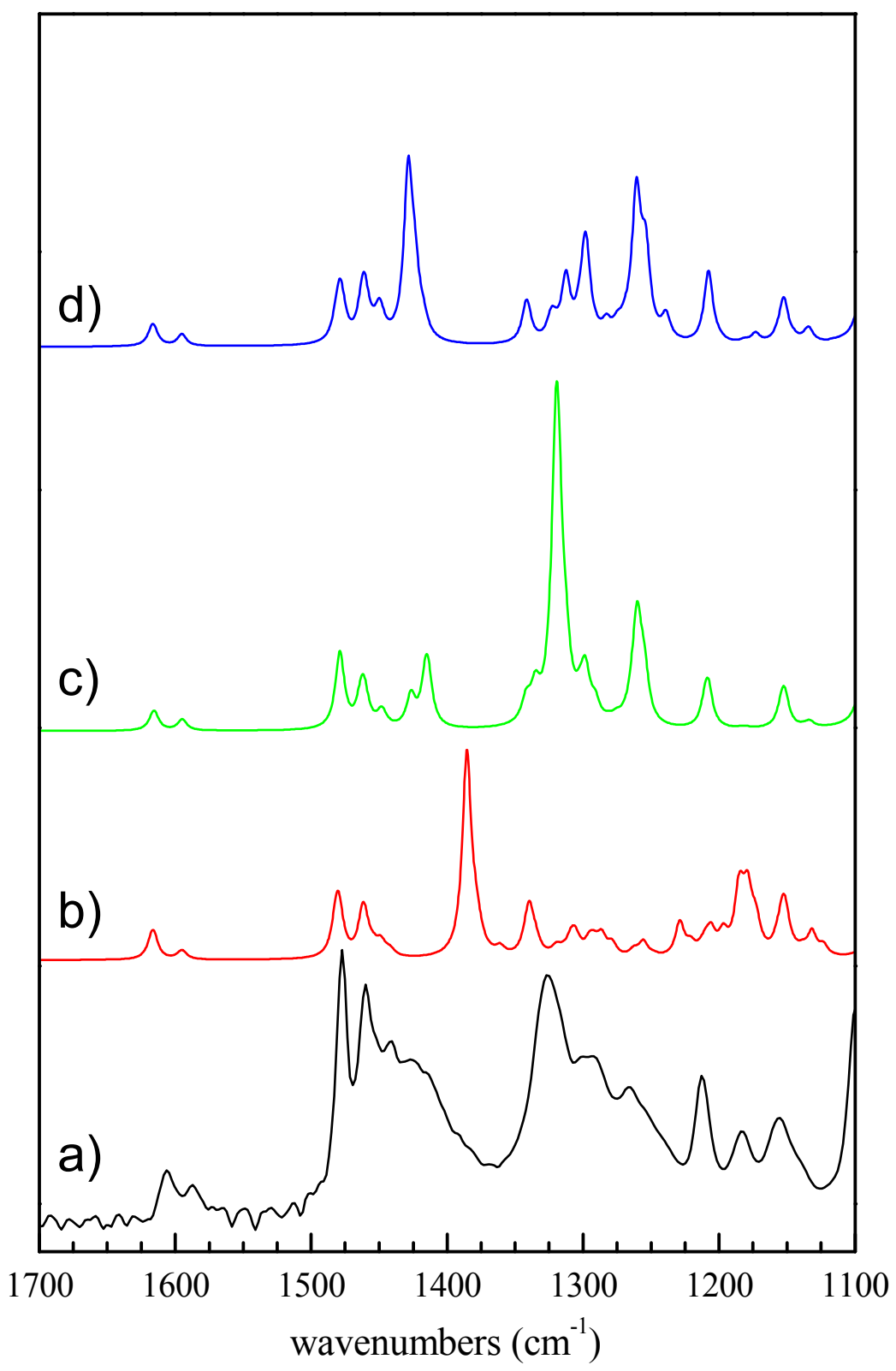

Figure 4: a) Experimental IR absorption spectrum of (S)-1-indanol in DMSO.

Simulated IR absorption spectrum obtained from the weighted IR absorption spectra of the most stable b) (S)-1-indanol conformers in a DMSO continuum c) (S)-1-indanol:DMSOd6 complexes in a DMSO continuum d) (S)-1-indanol:(DMSOd6) 2 complexes in a DMSO continuum, calculated at the B3LYP/6-31G++(d, p) level. 


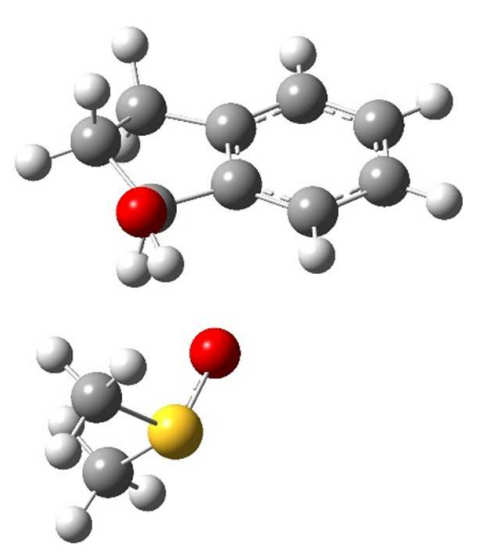

2eq-a : $40 \%$

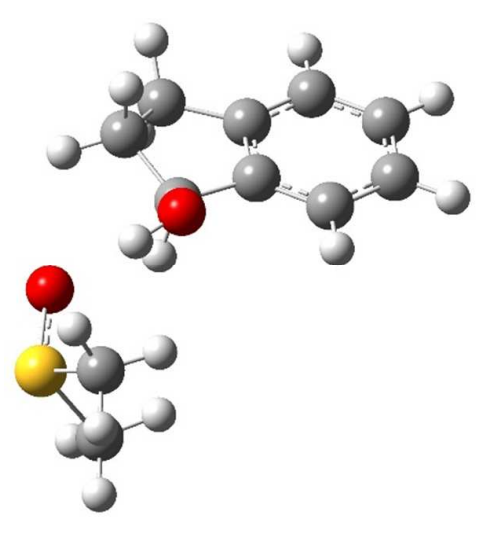

3eq : $34 \%$

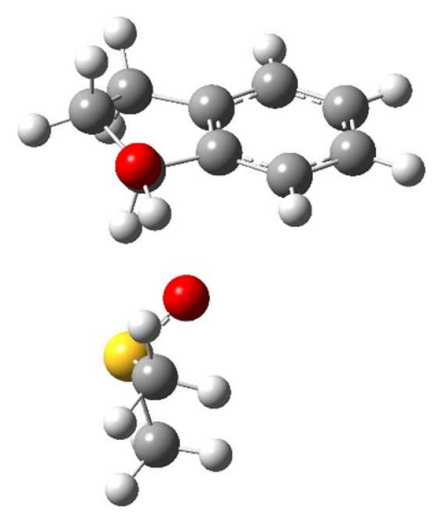

2 eq-b : $20 \%$

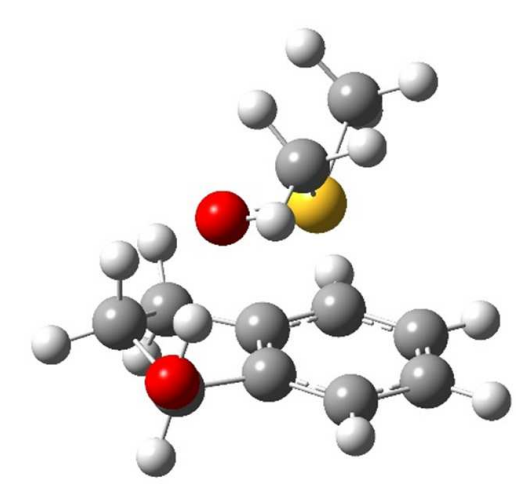

1eq : $6 \%$

Figure 5: Most stable structures of the (S)-1-indanol/DMSO complex in DMSO continuum calculated at the B3LYP/6-31++g(d,p) level. The The relative abundances are given in $\%$ of the total population of the (S)-1-indanol:DMSO complex. 


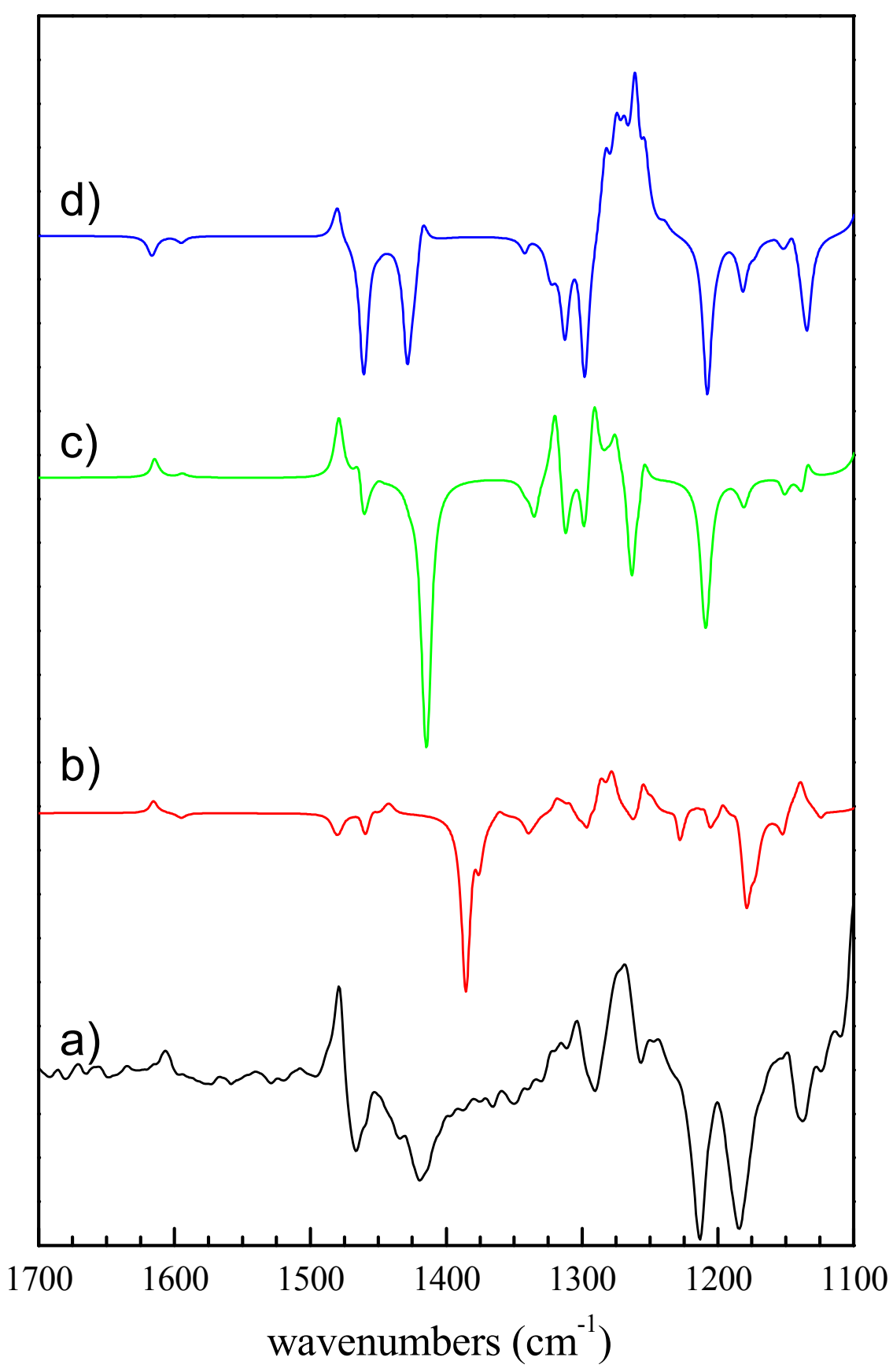

Figure 6: a) Experimental VCD spectrum of (S)-1-indanol in DMSO. Simulated VCD spectrum obtained from the weighted VCD spectra of the most stable b) (S)-1-indanol conformers in a DMSO continuum c) (S)-1-indanol:DMSO complexes in a DMSO continuum d) (S)-1-indanol:(DMSO $)_{2}$ complexes in a DMSO continuum, calculated at the B3LYP/6$31 \mathrm{G}++(\mathrm{d}, \mathrm{p})$ level. 


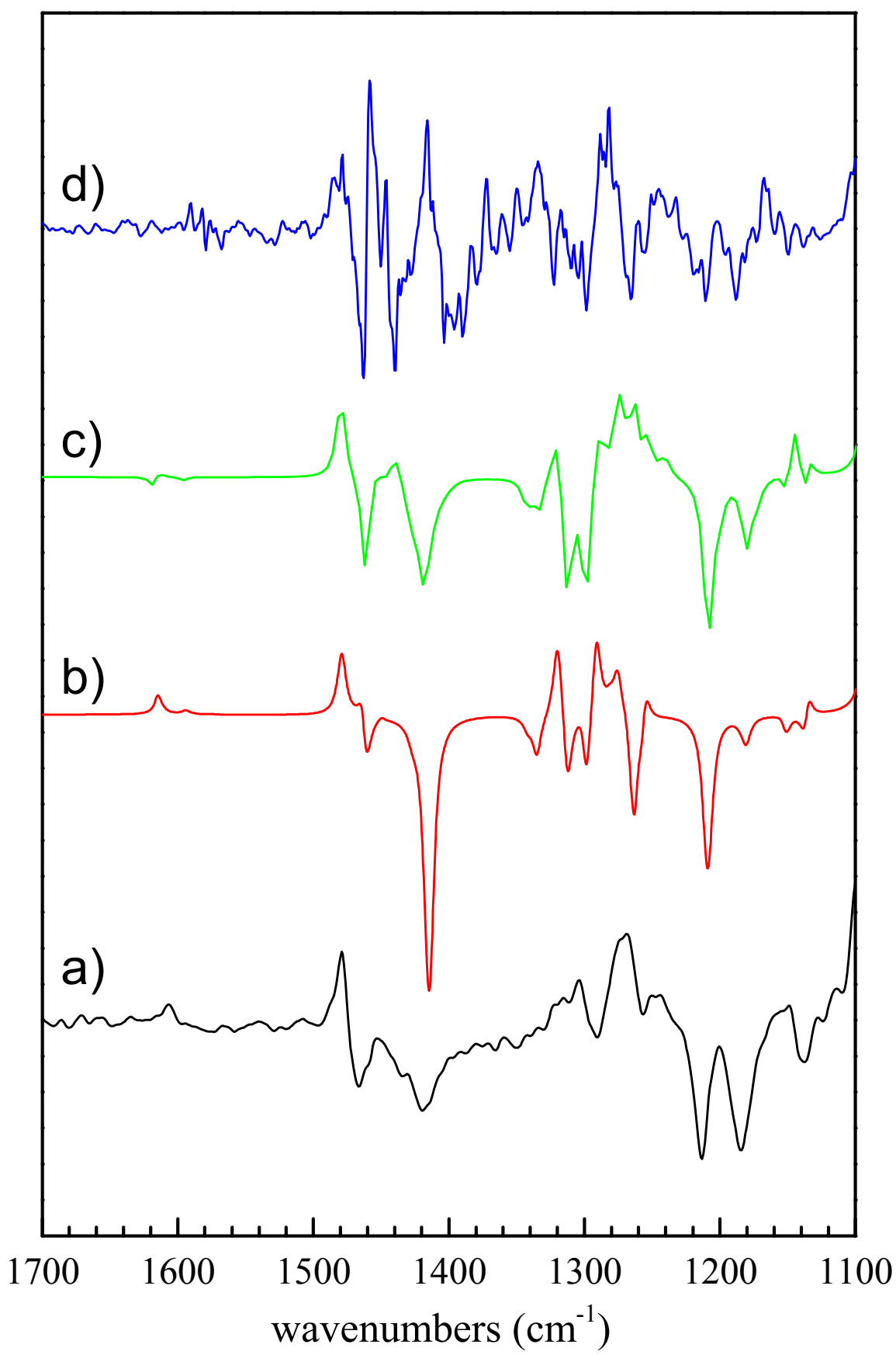

Figure 7: a) Experimental VCD spectrum of (S)-1-indanol in DMSO. Simulated VCD spectrum obtained from $b$ ) the weighted average of the VCD spectra of the most stable (S)-1indanol:DMSO complexes in a DMSO continuum calculated at the B3LYP/6-31G++(d, p) level c) the average of the VCD spectra of the 124 individual snapshots extracted from the NVT MD trajectory and further optimized at the B3LYP/6-31G++(d, p) level (see text) d) VCD spectrum simulated from the averaged analyses of two NVE trajectories (see text). 


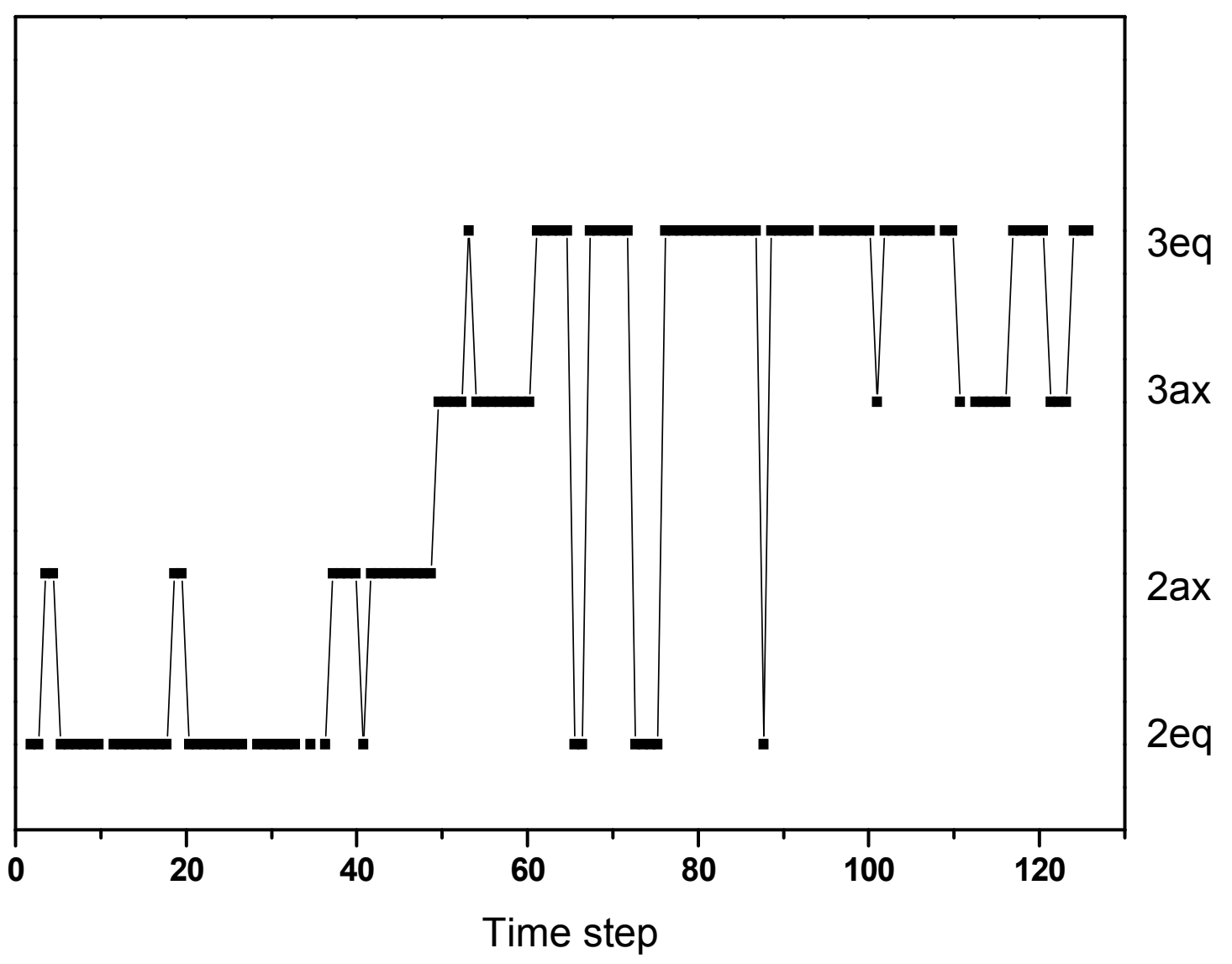

Figure 8: Evolution of the (S)-1-indanol geometry during the NVT FPMD simulation. Each time step corresponds to 0.25 ps. 


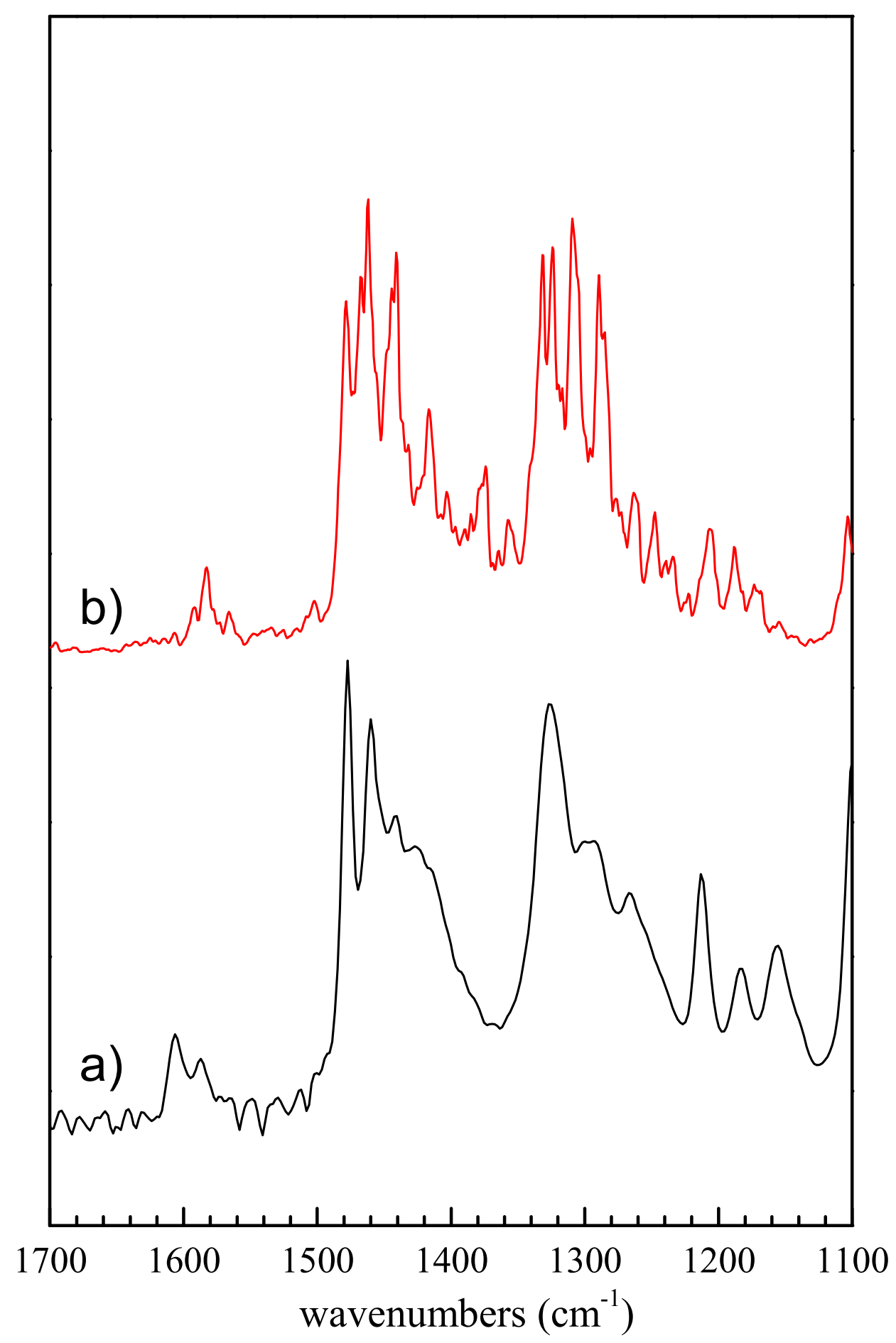

Figure 9: a) Experimental IR absorption spectrum of (S)-1-indanol in DMSO b) IR absorption spectrum simulated from the averaged analyses of two NVE trajectories (see text). 
a)

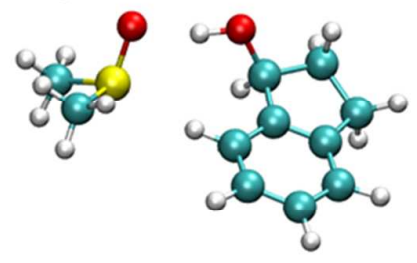

b)
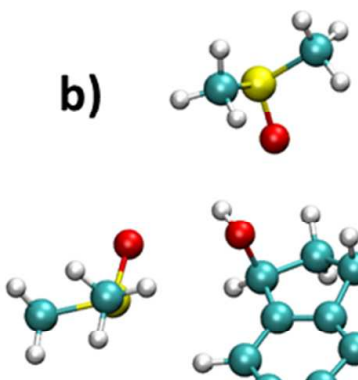

c)
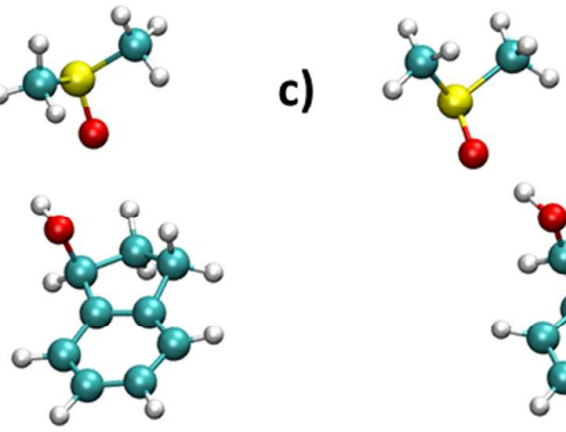

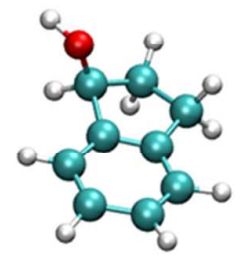

Figure 10: Snapshots of the (S)-1-indanol geometry along the MD NVT trajectory in DMSO a) a) $5000 \mathrm{fs}$ b) $10393 \mathrm{fs}$ c) $15000 \mathrm{fs}$

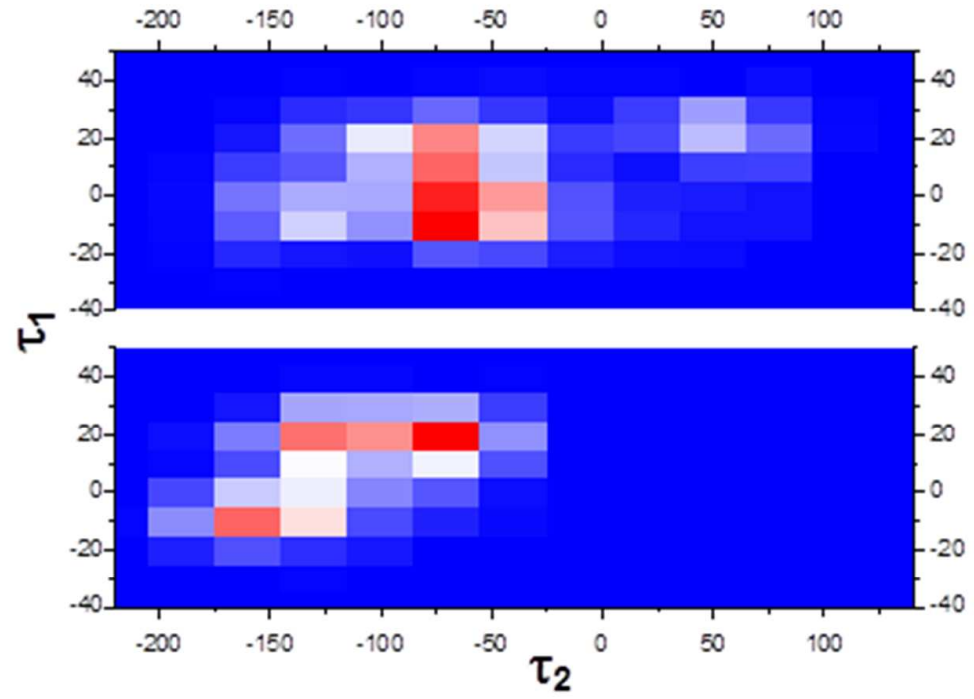

Figure 11. Evolution of the $\tau_{1}$ and $\tau_{2}$ angles during the NVE trajectories at $18 \mathrm{ps}$ (top) and 22 ps (bottom). The data are binned by 10 and $30^{\circ}$ for $\tau_{1}$ and $\tau_{2}$, respectively and divided in 10 bins with thermometer false colour range, from deep blue for bin with zero population to scarlet for the most populated bin. 


\title{
Effect of Puckering Motion and Hydrogen Bond Formation on the
} Vibrational Circular Dichroism Spectrum of a Flexible Molecule: the case of (S)-1-Indanol

\author{
Katia Le Barbu-Debus, ${ }^{\text {a) }}$ Arne Scherrer, ${ }^{\text {b) c) }}$ Aude Bouchet, ${ }^{\text {a) }}$ \\ Daniel Sebastiani, ${ }^{\text {c) }}$ Rodolphe Vuilleumier, ${ }^{*}$ b) Anne Zehnacker, * a) \\ a) Institut des Sciences Moléculaires d'Orsay (ISMO), CNRS, Univ. Paris-Sud, \\ Université Paris-Saclay, F-91405 Orsay (France). \\ b) PASTEUR, Département de chimie, École normale supérieure, PSL University, \\ Sorbonne Université, CNRS, 75005 Paris, France \\ c) Martin-Luther-Universität Halle-Wittenberg, Institut für Chemie, von-Danckelmann- \\ Platz 4, 06120 Halle, Germany \\ *e-mail: anne.zehnacker-rentien@u-psud.fr, rodolphe.vuilleumier@ens.fr
}

\section{Supplementary Information}


Table S1 Energetic and structural parameters of the most stable conformers of (S)-1-indanol in a $\mathrm{CCl}_{4}$ continuum calculated at the BLYP/6-31G++(d,p) level

\begin{tabular}{|c|c|c|c|c|}
\hline Geometry & $\begin{array}{l}\text { Gibbs free } \\
\text { energy } \\
(\mathrm{kcal} / \mathrm{mol})\end{array}$ & $\begin{array}{c}\text { Boltzmann } \\
\text { population } \\
(\%)\end{array}$ & $\begin{array}{c}\mathrm{C}_{9} \mathrm{C}_{4} \mathrm{C}_{3} \mathrm{C}_{2} \\
\text { angle } \\
\left(^{\circ}\right)\end{array}$ & $\begin{array}{c}\mathrm{C}_{9} \mathrm{C}_{1} \mathrm{OH} \\
\text { angle } \\
\left({ }^{\circ}\right)\end{array}$ \\
\hline $1 \mathrm{eq}$ & 0.00 & 28 & 16.4 & 55.1 \\
\hline $1 \mathrm{ax}$ & 0.16 & 22 & -12.7 & 44.9 \\
\hline $2 a x$ & 0.64 & 10 & -14.2 & -54.6 \\
\hline $3 \mathrm{eq}$ & 0.56 & 11 & 16.0 & -167.4 \\
\hline $2 \mathrm{eq}$ & 0.21 & 20 & 15.7 & -77.4 \\
\hline $3 a x$ & 0.67 & 9 & -14.7 & -176.0 \\
\hline
\end{tabular}

Table S2 Energetic and structural parameters of the most stable conformers of (S)-1-indanol in a DMSO continuum calculated at the B3LYP-D3/6-31G++ $(\mathrm{d}, \mathrm{p})$ level

\begin{tabular}{|c|c|c|c|c|}
\hline Geometry & $\begin{array}{l}\text { Gibbs free } \\
\text { energy } \\
\text { (kcal/mol) }\end{array}$ & $\begin{array}{c}\text { Boltzmann } \\
\text { population } \\
(\%)\end{array}$ & $\begin{array}{c}\mathrm{C}_{9} \mathrm{C}_{4} \mathrm{C}_{3} \mathrm{C}_{2} \\
\text { angle } \\
\left({ }^{\circ}\right)\end{array}$ & $\begin{array}{c}\mathrm{C}_{9} \mathrm{C}_{1} \mathrm{OH} \\
\text { angle } \\
\left({ }^{\circ}\right)\end{array}$ \\
\hline $1 \mathrm{eq}$ & 0.00 & 35 & 17.4 & 54.9 \\
\hline $1 a x-a$ & 0.05 & 31 & -13.7 & 54.6 \\
\hline $1 a x-b$ & 0.46 & 16 & -15.5 & 48.7 \\
\hline $1 \mathrm{ax}-\mathrm{c}$ & 0.57 & 13 & -16.8 & 55.0 \\
\hline $2 a x$ & 1.16 & 5 & -15.6 & -44.9 \\
\hline
\end{tabular}




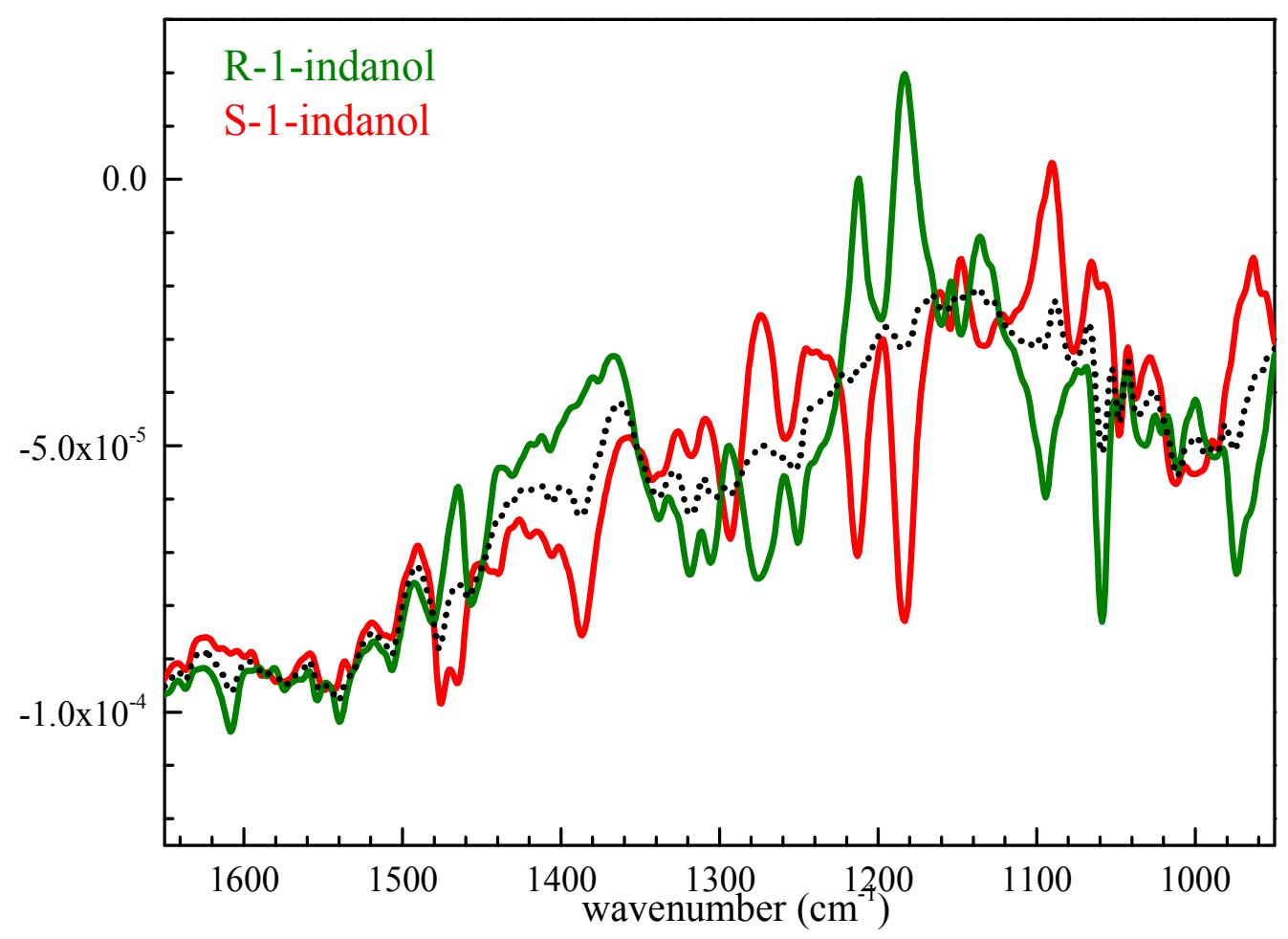

Figure S1: Raw experimental VCD spectra of R and S-1-Indanol together with the baseline (dotted line). 


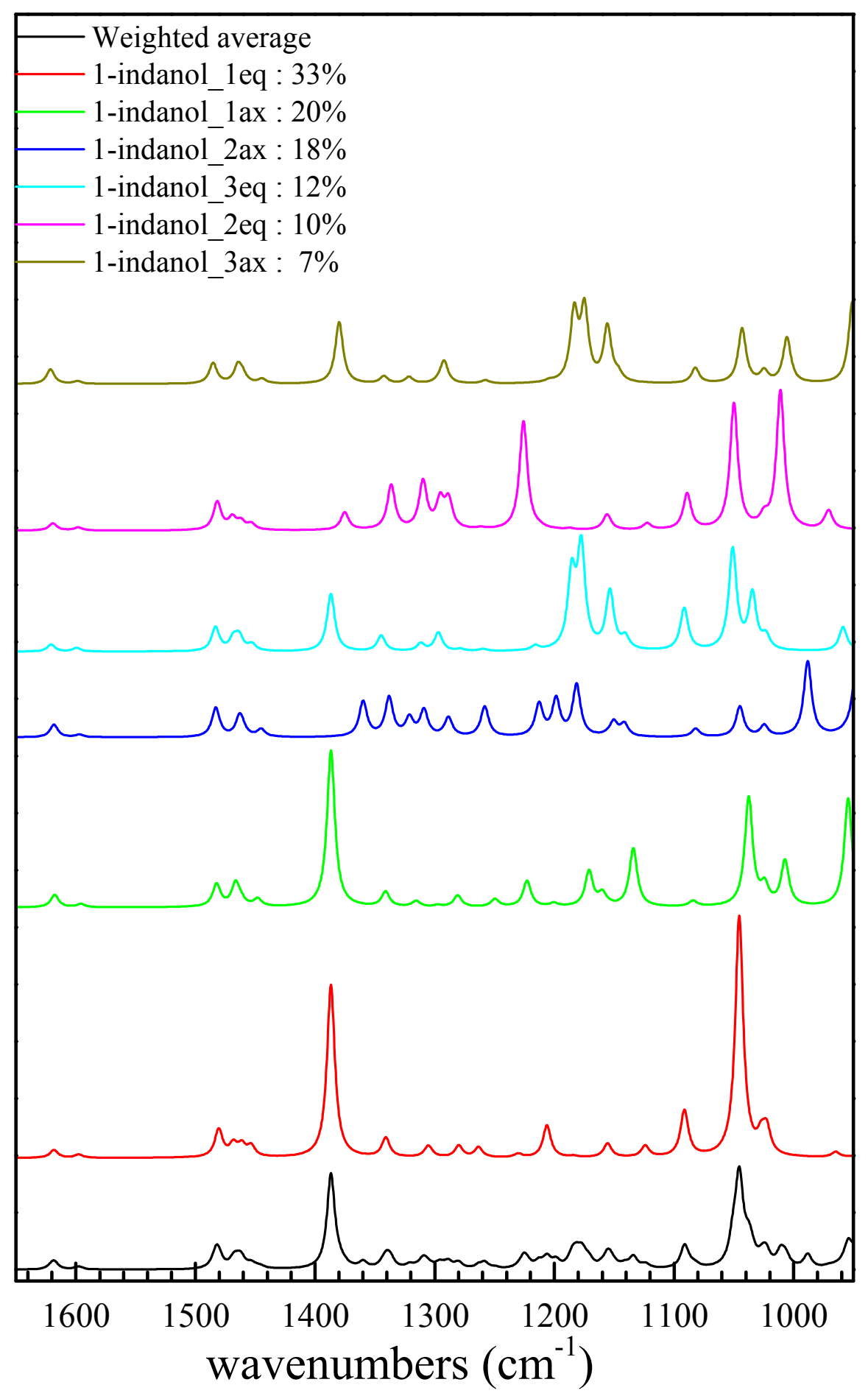

Figure S2: Simulated IR spectrum obtained from the weighted IR spectra of the most stable (S)-1-indanol structures in a $\mathrm{CCl}_{4}$ continuum together with individual contributions, calculated at the B3LYP/6-31G++(d, p) level 


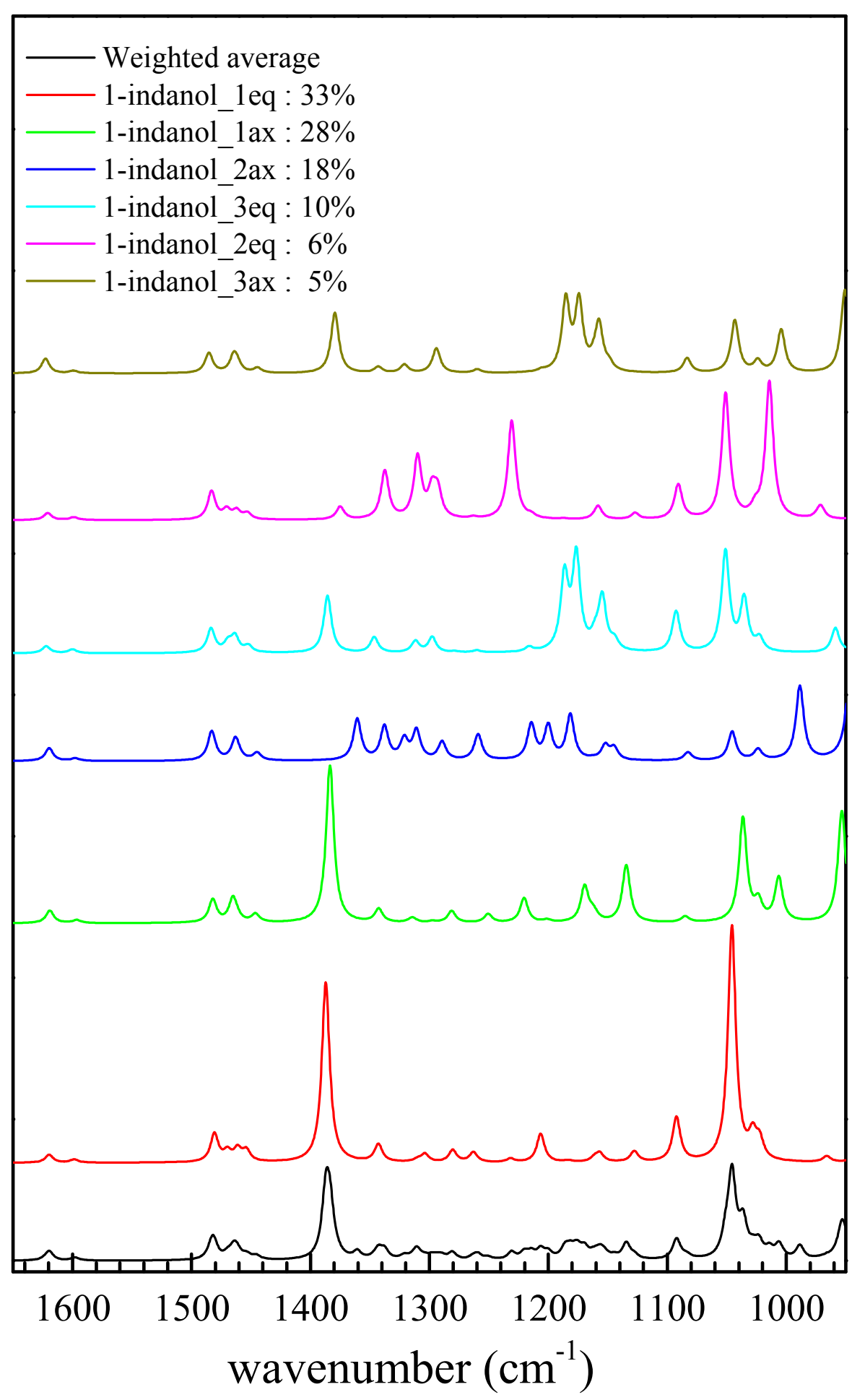

Figure S3: Simulated IR spectrum obtained from the weighted IR spectra of the most stable 1(S)-1-indanol structures in a $\mathrm{CCl}_{4}$ continuum together with individual contributions, calculated at the B3LYP-D3/6-31G++(d, p) level 


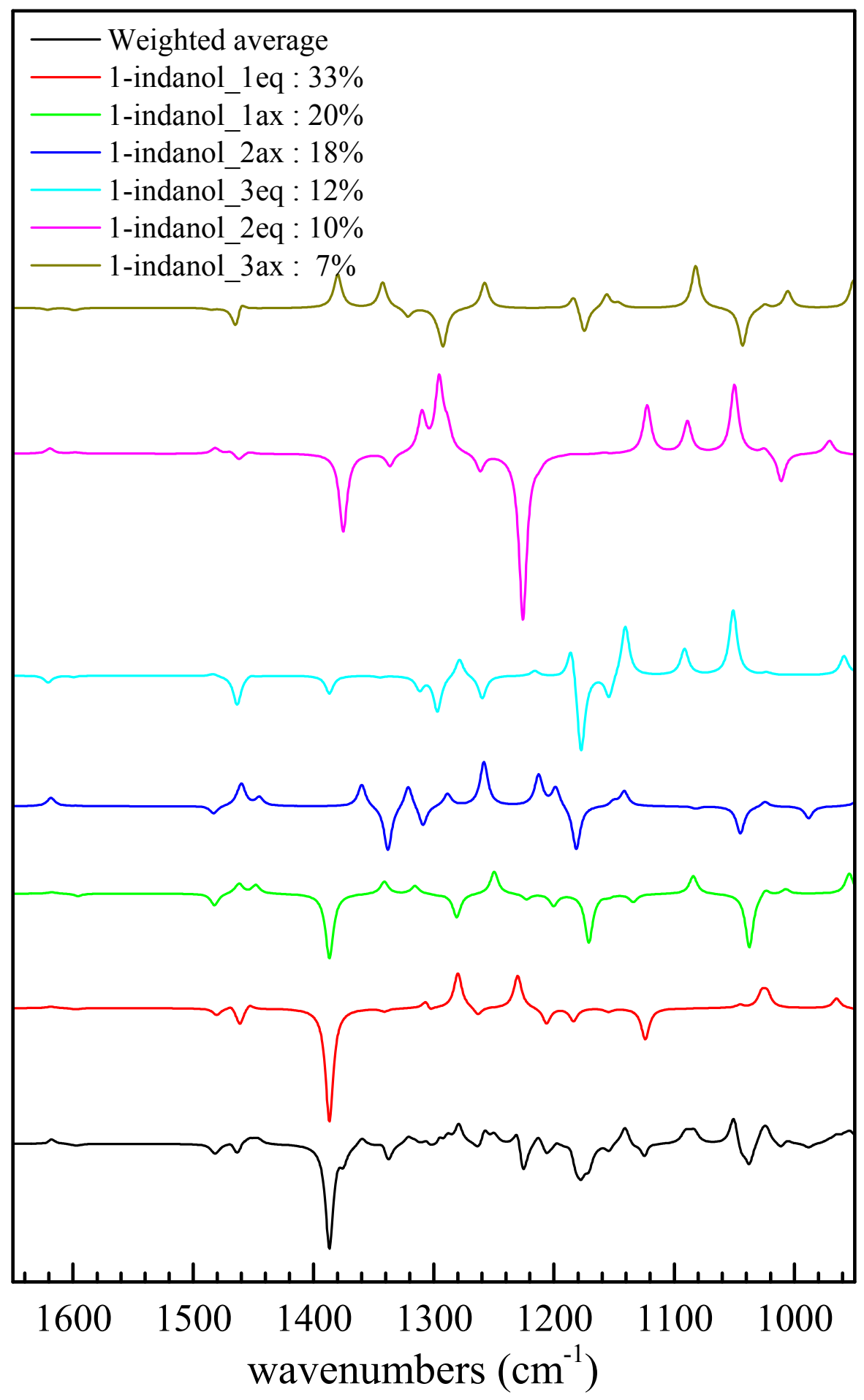

Figure S4: Simulated VCD spectrum obtained from the weighted VCD spectra of the most stable (S)-1-indanol structures in a $\mathrm{CCl}_{4}$ continuum together with individual contributions, calculated at the B3LYP/6-31G++(d, p) level 


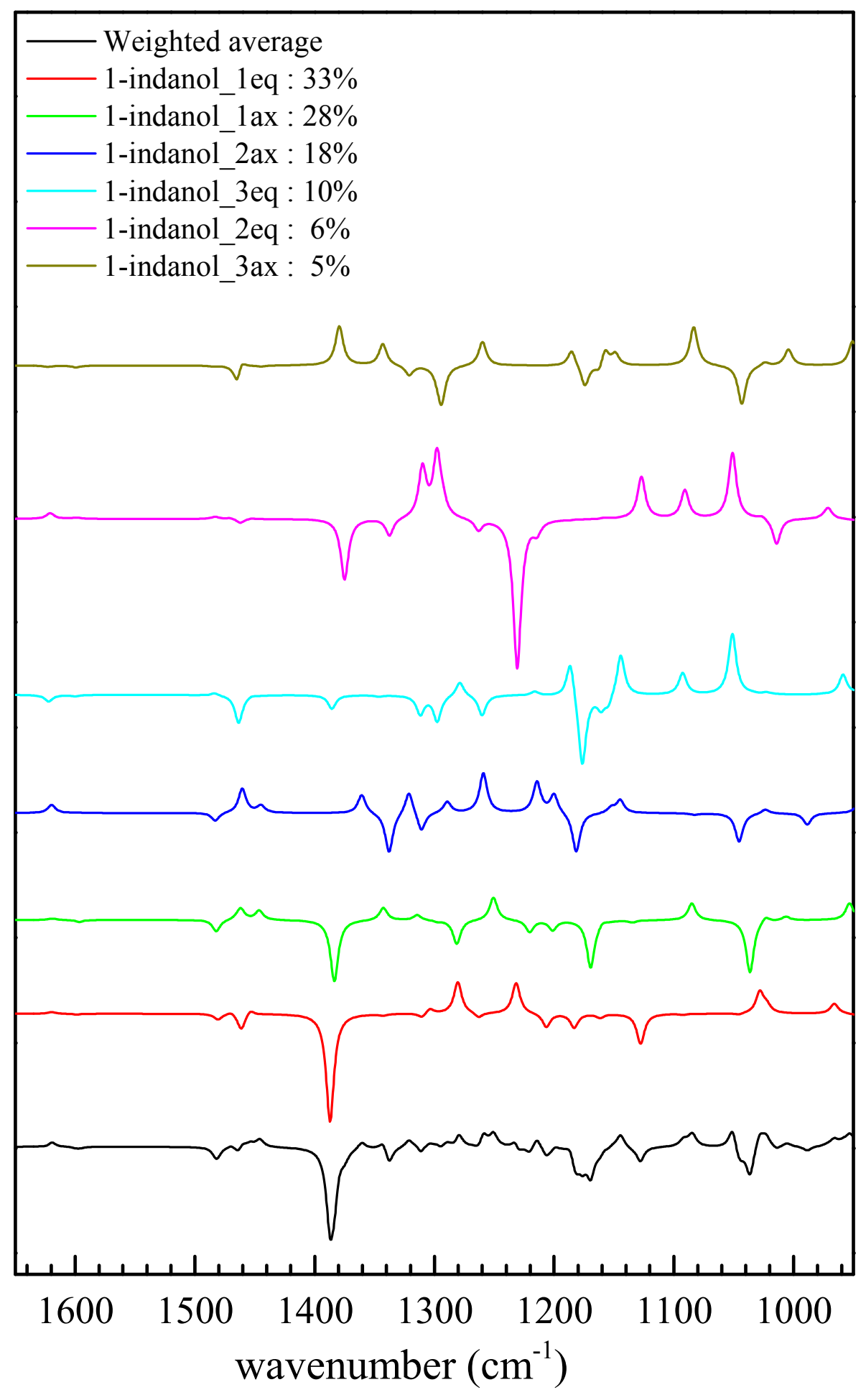

Figure S5: Simulated VCD spectrum obtained from the weighted VCD spectra of the most stable (S)-1-indanol structures in a $\mathrm{CCl}_{4}$ continuum together with individual contributions, calculated at the B3LYP-D3/6-31G++(d, p) level 


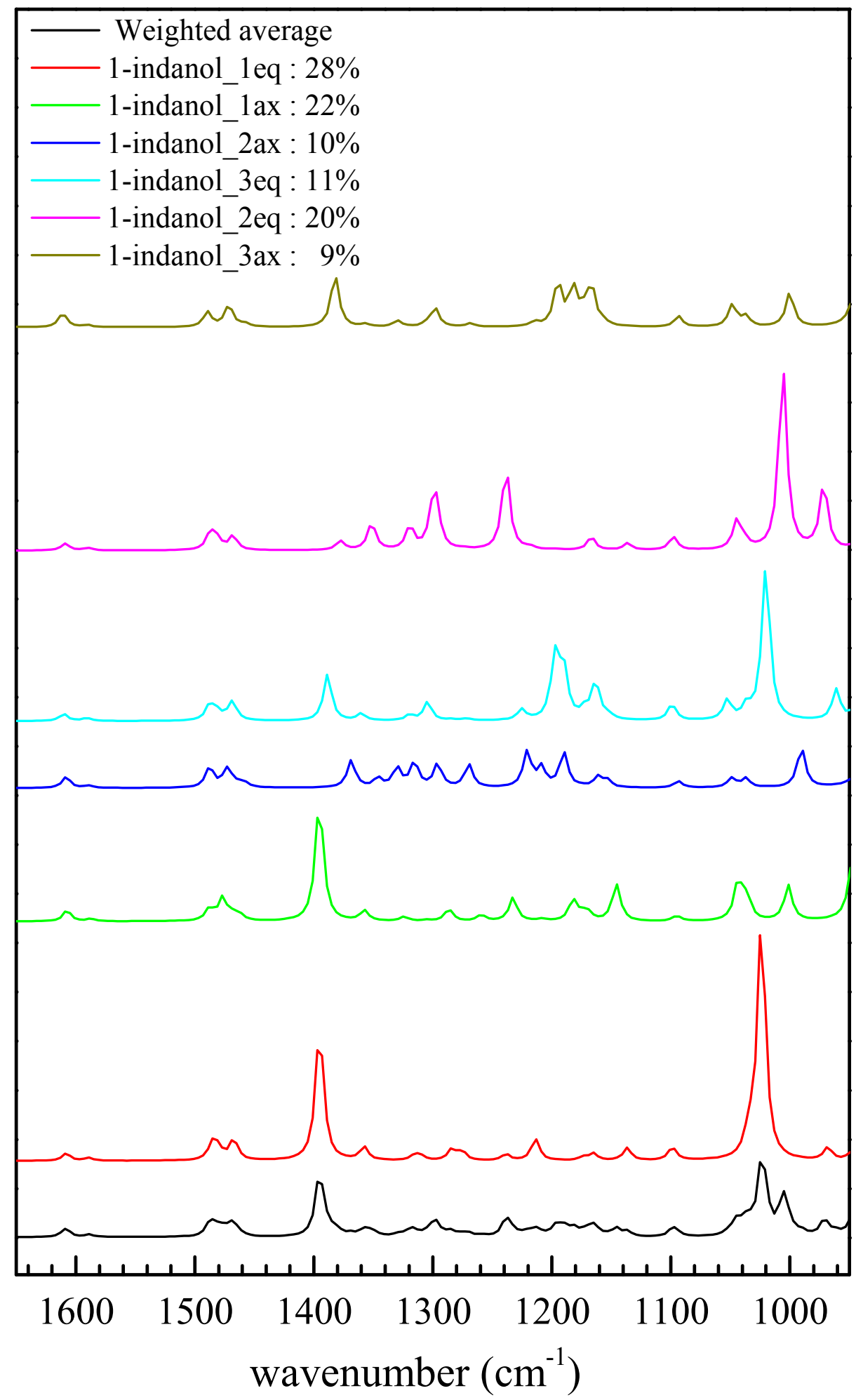

Figure S6: Simulated IR spectrum obtained from the weighted IR spectra of the most stable

(S)-1-indanol structures in a $\mathrm{CCl}_{4}$ continuum together with individual contributions, calculated at the BLYP/6-31G++(d, p) level. 


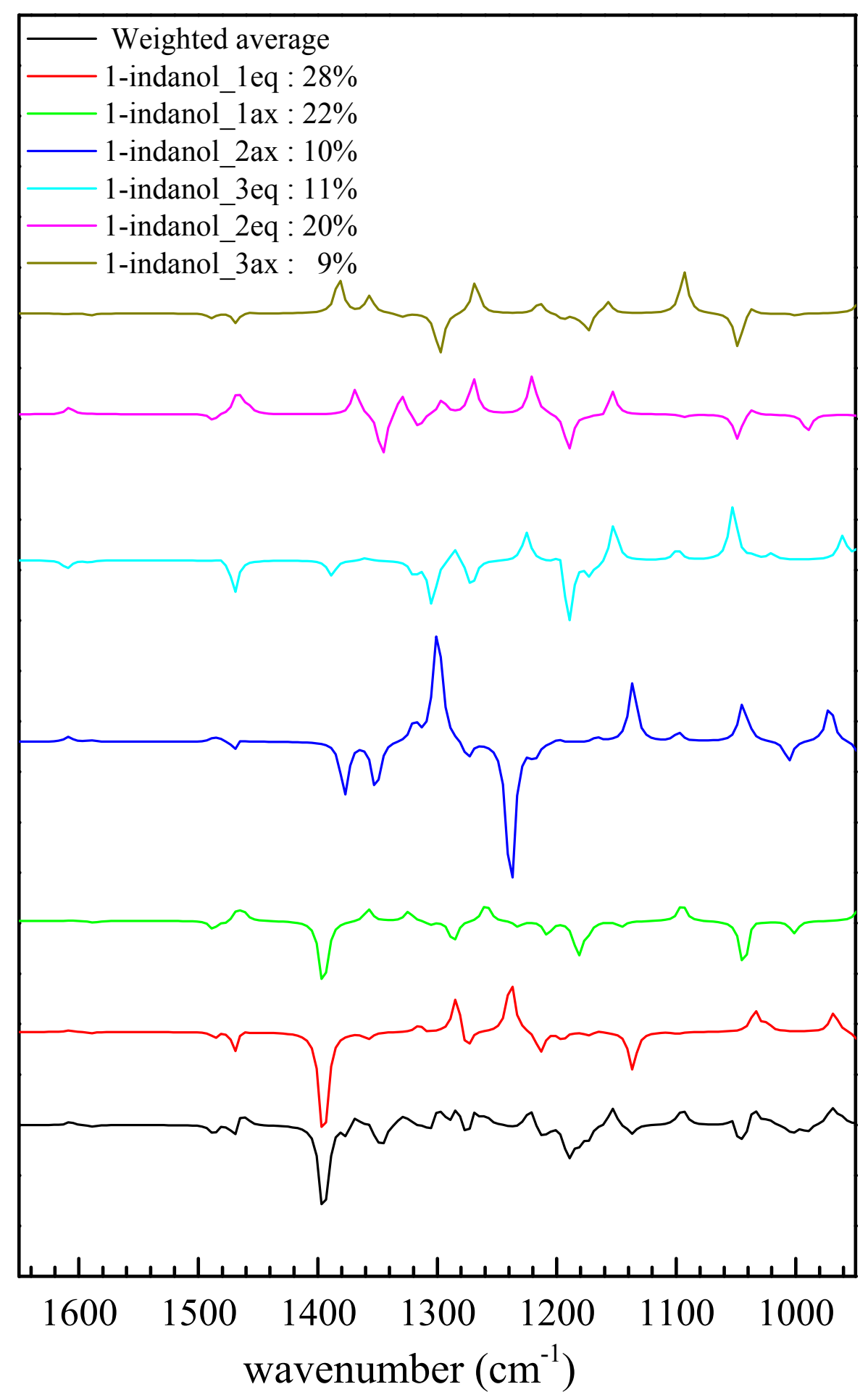

Figure S7: Simulated VCD spectrum obtained from the weighted VCD spectra of the most stable (S)-1-indanol structures in a $\mathrm{CCl}_{4}$ continuum together with individual contributions, calculated at the BLYP/6-31G++(d, p) level. 


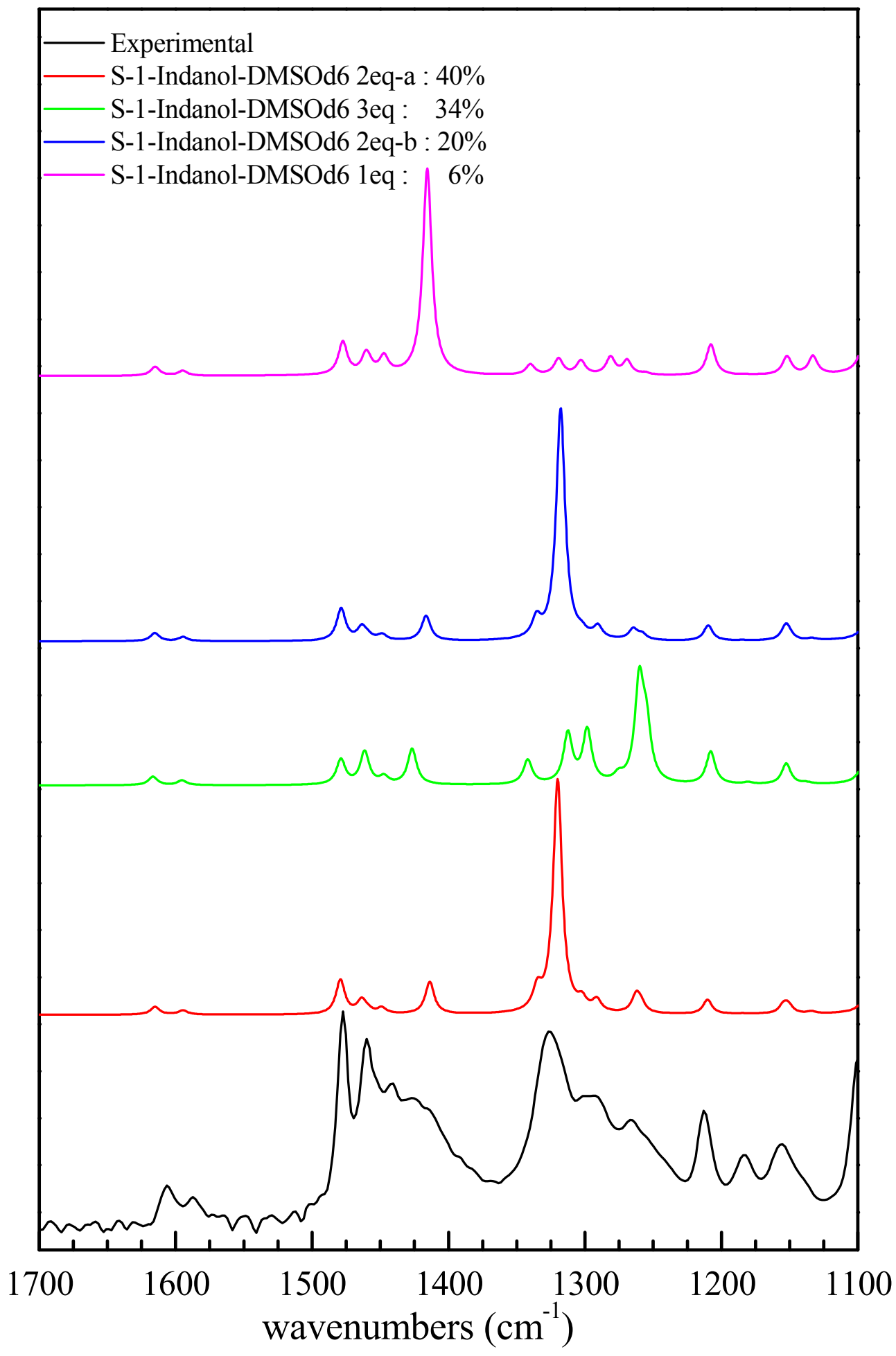

Figure S8: Simulated IR spectra of the most stable (S)-1-indanol:DMSO-d6 complexes in a DMSO continuum, calculated at the B3LYP/6-31G $++(d, p)$ level, together with experimental spectrum. 


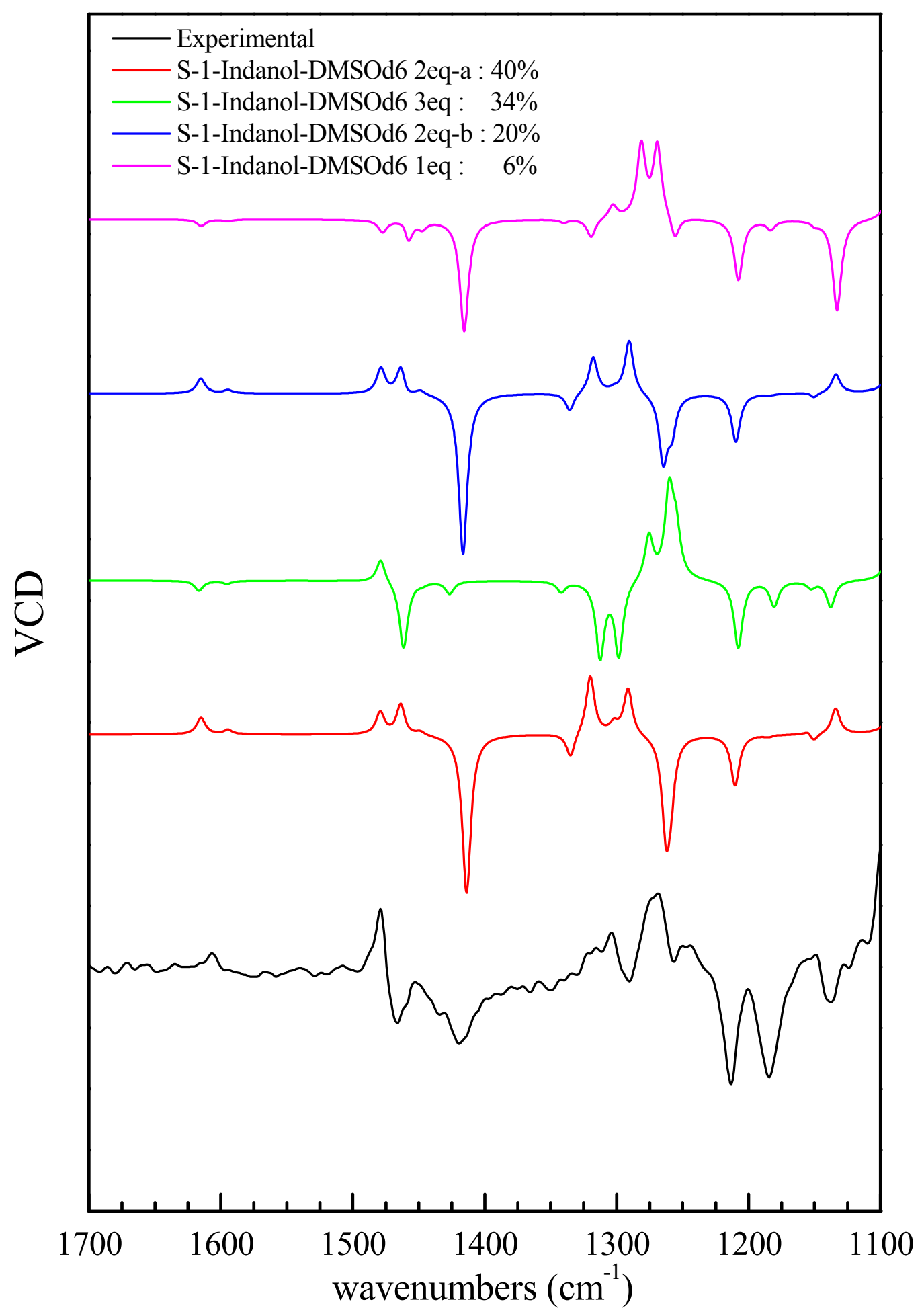

Figure S9: Simulated VCD spectra of the most stable (S)-1-indanol:DMSO-d6 complexes in a DMSO continuum, calculated at the B3LYP/6-31G $++(d, p)$ level, together with experimental spectrum 


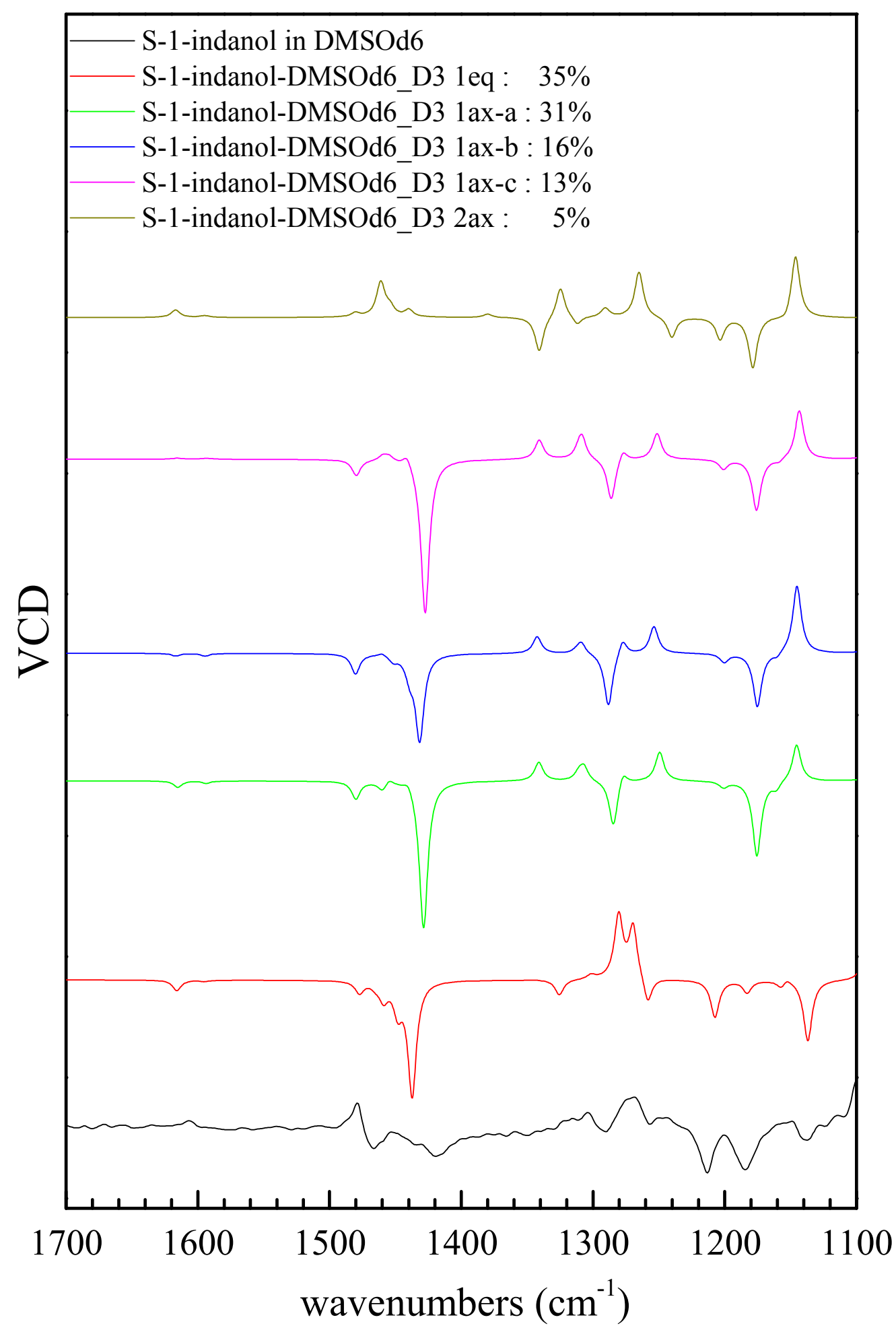

Figure S10: Simulated VCD spectra of the most stable (S)-1-indanol:DMSO-d6 complexes in a DMSO continuum, calculated at the B3LYP-D3/6-31G++(d,p) level, together with experimental spectrum. 

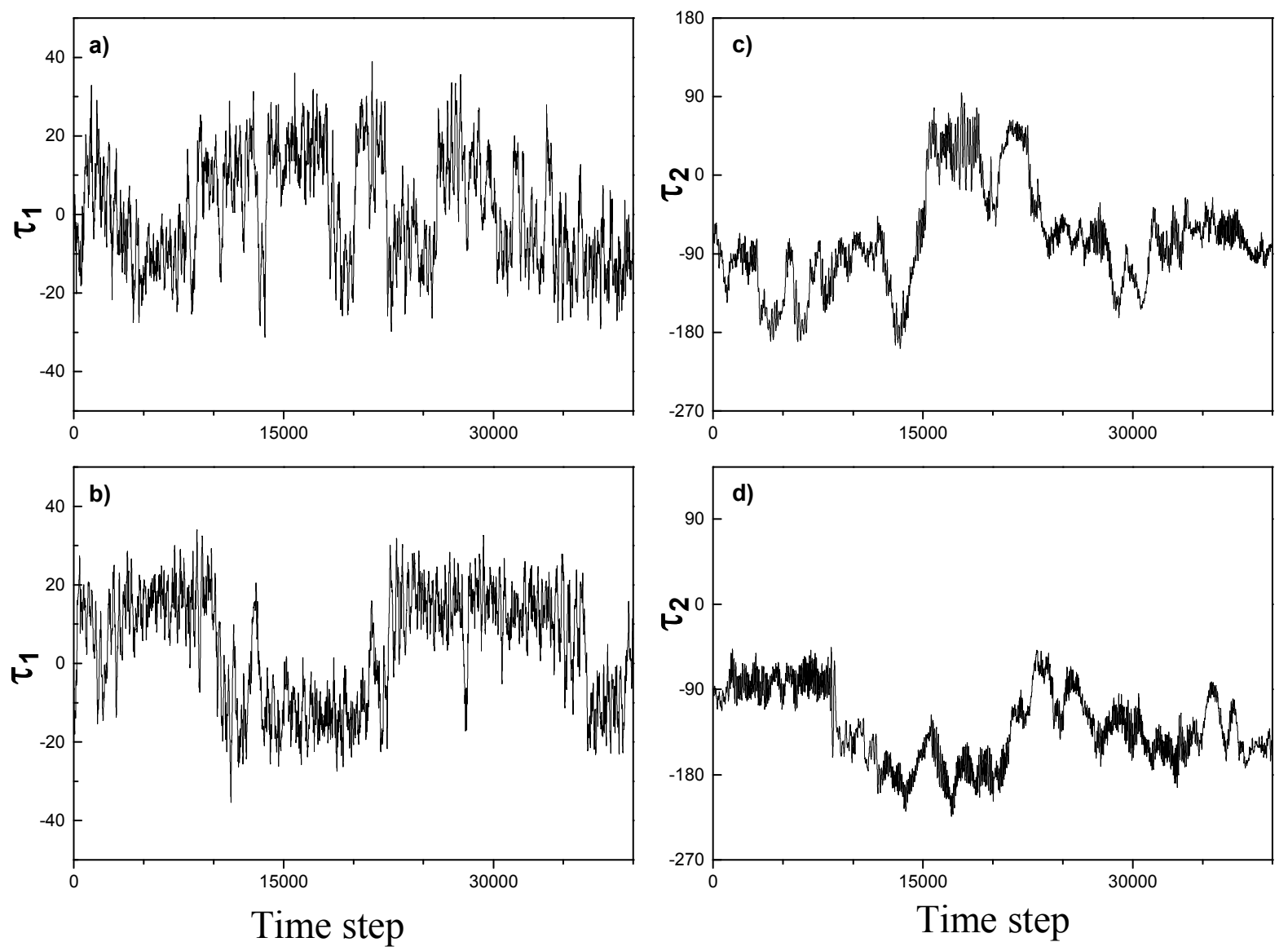

Figure S11. Evolution of the $\tau_{1}$ and $\tau_{2}$ angles during the NVE trajectories. a) $\tau_{1}$ and b) $\tau_{2}$ angles for the trajectory at $18 \mathrm{ps} \mathrm{c)} \tau_{1}$ and d) $\tau_{2}$ angles for the trajectory at $22 \mathrm{ps}$. 